\title{
Nonlinear dynamics of slender inverted flags in uniform steady flows
}

\author{
Mohammad Tavallaeinejad, Mathias Legrand, Michael Païdoussis \\ Department of Mechanical Engineering, McGill University, Montréal, Québec, Canada H3A 0C3
}

\begin{abstract}
A nonlinear fluid-elastic continuum model for the dynamics of a slender cantilevered plate subjected to axial flow directed from the free end to the clamped end, also known as the inverted flag problem, is proposed. The extension of elongated body theory to large-amplitude rotations of the plate mid-plane along with Bollay's nonlinear wing theory is employed in order to express the fluid-related forces acting on the plate, while retaining all time-dependent terms in both modelling and numerical simulations; the unsteady fluid forces due to vortex shedding are not included. Euler-Bernoulli beam theory with exact kinematics and inextensibility is employed to derive the nonlinear partial integro-differential equation governing the dynamics of the plate. Discretization in space is carried out via a conventional Galerkin scheme using the linear mode-shapes of a cantilevered beam in vacuum. The pseudo-arclength continuation technique is adapted to construct bifurcation diagrams in terms of the flow velocity, in order to gain insight into the stability and post-critical behaviour of the system. Integration in time is conducted using Gear's backward differentiation formula. The sensitivity of the nonlinear response of the system to different parameters such as the aspect ratio, mass ratio, initial inclination of the flag, and viscous drag coefficient is investigated through extensive numerical simulations. It is shown that for flags of small aspect ratio the undeflected static equilibrium is stable prior to a subcritical pitchfork bifurcation. For flags of sufficiently large aspect ratio, however, the first instability encountered is a supercritical Hopf bifurcation giving rise to flapping motion around the undeflected static equilibrium; increasing the flow velocity further, the flag then displays flapping motions around deflected static equilibria, which later lead to fully-deflected static states at even higher flow velocities. The results exposed in this study help understand the dynamics of the inverted-flag problem in the limit of inviscid flow theory.
\end{abstract}

Keywords inverted flag, bistability, nonlinear dynamics, fluid-structure interactions

\section{Nomenclature}

$\begin{array}{ll}H, L, h & \text { Flag height/length/thickness } \\ A & \text { Flag cross-sectional area } \\ I & \text { Flag second moment of inertia } \\ D & \text { Flag flexural rigidity } \\ \rho_{\mathrm{p}}, \rho_{\mathrm{f}} & \text { Flag/fluid mass density } \\ \eta & \text { Material viscosity coefficient } \\ v & \text { Poisson's ratio } \\ U & \text { Free-stream fluid flow velocity } \\ c_{\mathrm{d}} & \text { External viscous damping coefficient } \\ v_{\mathrm{f}} & \text { Fluid kinematic viscosity } \\ f, T & \text { Frequency/period of oscillation } \\ R & \text { Flag aspect ratio } \\ \mu & \text { Mass ratio } \\ \tau & =\sqrt{\rho_{\mathrm{p}} A / D} L^{2} \\ \beta & =I /\left(A L^{2}\right) \\ R e & \text { Reynolds number } \\ \Pi & \text { Dimensionless flow velocity } \\ t, x & \text { Time and space parametrization } \\ u(x, t) & \text { Longitudinal in-plane deflection } \\ w(x, t) & \text { Transverse deflection } \\ \alpha(x, t) & \text { Instantaneous angle of attack }\end{array}$

$\begin{array}{ll}\theta(x, t) & \text { Angle of relative flow velocity } \\ \psi(x, t) & \text { Mid-plane slope = cross-section rotation } \\ \psi_{0} & \text { Incidence angle } \\ \phi_{r}(x), q_{r}(t) & r \text { th modeshape/generalized coordinate } \\ F_{\mathrm{N}}(x, t) & \text { Reactive component of fluid forces } \\ F_{\mathrm{R}}(x, t) & \text { Nonlinear vortex lift } \\ F_{\tau}(x, t) & \text { Tangential viscous drag force } \\ K_{\mathrm{p}}(R) & \text { Normal force coefficient due to circulation } \\ K_{\mathrm{v}}(R) & \text { Normal force coefficient due to side-edge } \\ & \text { vortex flow } \\ K_{\tau}(R e) & \text { Drag coefficient due to viscous stresses } \\ V_{n}(x, t) & \text { Relative velocity normal to the centreline } \\ V_{\tau}(x, t) & \text { Relative velocity parallel to the centreline } \\ \mathcal{T}(t), \mathcal{V}(t) & \text { Flag kinetic/potential energy } \\ W_{\mathrm{f}}(t), W_{\mathrm{d}}(t) & \text { Virtual work of fluid-forces/damping } \\ \partial_{t} \bullet,[\bullet]_{t},: & \text { First time derivative } \\ \partial_{x} \bullet,[\bullet]_{x} & \text { First space derivative } \\ \partial_{t t},[\bullet]_{t t}, \bullet & \text { Second time derivative } \\ {[\bullet]_{x x}, \bullet x x} & \text { Second space derivative } \\ \bullet{ }_{t x x} & \text { First time and second space derivative } \\ \bullet * & \text { Dimensionless counterpart of } \bullet\end{array}$

\section{Introduction}

The dynamics of cantilevered plates in incompressible reverse axial flows, otherwise known as inverted flags, is of considerable interest, not only as an abstract problem with rich dynamics, but also for engineering applications such as small scale energy harvesting systems [19, 28] and heat transfer enhancement in heat exchangers [35, 20]. Generally speaking, an inverted flag may lose stability at a sufficiently high flow velocity and exhibit a flapping 
motion. A familiar example is the flapping motion of leaves in the wind. There has been ongoing research on this fluid-structure interaction problem over the past few years, with the aim of understanding the essential dynamics. These studies may be classified into three categories: experimental investigations, computational fluid dynamics (CFD) analyses utilizing numerical solvers for the Navier-Stokes equations, and analytical studies using simplified continuum expressions for the aerodynamic forces.

Starting with analytical and experimental studies, a linear stability analysis was first carried out by Guo \& Païdoussis [12] who employed inviscid potential flow theory to predict the onset of oscillatory instability. They showed that free-clamped plates (inverted flags) flutter at critical velocities inversely proportional to their mass ratios $^{1}$. The flapping motion of inverted flags subjected to uniform air and water flows was explored in the pioneering experimental work of Kim et al. [15]. Three regimes were reported as the flow velocity was varied: the straight (undeflected) stationary regime, the flapping regime with large amplitude, and a fully deflected static regime. Bistable states were also observed in the transition from straight-to-flapping and flapping-to-deflected regimes.

This work was pursued by Sader et al. [24, 25] who conducted experiments on inverted flags of various aspect ratios. Their experimental observations revealed that the aspect ratio of a flag can dramatically alter the dynamics. In particular, slender flags exhibit a buckling behaviour via a static divergence instability. Intermediately wide flags, on the other hand, undergo an abrupt flapping motion prior to a fully deflected regime, with a highly curved shape, via buckling. Moreover, they reported that the undeflected static equilibrium of inverted flags with smaller aspect ratios loses stability at higher critical flow velocities. In their first study, in conjunction with mathematical modelling based on a linear aerodynamic theory and a scaling analysis, Sader et al. [24] concluded that flapping motion of inverted flags subjected to heavy fluid loading (light flags) is a vortex-induced vibration (VIV). For light fluid loading (heavy flags), however, the inverted flag cannot undergo a VIV, despite displaying large-amplitude flapping; the comprehension of the mechanism involved requires further work. In their second study, in addition to experimental measurements, Sader et al. [25] undertook a nonlinear theoretical investigation and provided an analytical framework to explore the stability and dynamics of inverted flags in the asymptotic limit of zero aspect ratio, within the restricted framework of steady fluid forces. Moreover, they formulated a mathematical solution to predict the onset of the static bifurcation for inverted flags with various aspect ratios. More specifically, they employed a Padé approximant to correlate the two solutions for zero-aspect-ratio and two-dimensional flow limits, yielding a formula applicable over the entire range of aspect ratios studied; a very good quantitative agreement with measurements was found. They offered a reasonable qualitative explanation that the deflected shape of the flag, originating from the the static divergence point, presents a sharp leading edge to the impinging flow, and the resulting unsteady vortex shedding causes the flag to flap. This analysis was subsequently extended by the authors [31] to deal with intermediate aspect ratios, as well as asymmetric behaviour of inverted flags in the presence of an initial curvature along the plate.

All analytical models to date are restricted to steady-flow theories, and do not provide any mathematical formulation or numerical framework to explore the effect of time-dependent reactive forces. Such modelling disallows the possibility of capturing flapping motion, which is a feature of inverted flags of sufficiently large aspect ratio. The need of modelling such effects has motivated the present study.

From the computational fluid dynamics perspective, various investigations have contributed to the field. For instance, Tang et al. [29] performed a three-dimensional (3D) simulation using an immersed boundary latticeBoltzmann approach focusing on low Reynolds number flows. They concluded that wider plates oscillate with a larger amplitude and undergo flapping motion at lower critical flow velocities. They also found that the stretchedstraight state of lighter plates is more stable. The effect of an initial angle of attack was also explored and it was shown that an asymmetric flapping regime emerges, which becomes narrower with an increasing angle of attack. In another study, Shoele and Mittal [28] investigated the energy-harvesting performance of inverted piezoelectric flags using an immersed boundary method. They also examined the effect of the initial inclination of an inverted flag to the impinging flow and showed that the dynamic response of the system is relatively insensitive to small deviations in the initial inclination angle. Gurugubelli and Jaiman [14] employed a second-order scheme combining the finite element procedure with the arbitrary Lagrangian-Eulerian (ALE) approach to conduct a two-dimensional (2D) numerical simulation on the inverted flag problem and found that the mass ratio affects the transition from large-amplitude flapping motion to moderate flapping around the deflected position of the inverted flag. In another study [13], they performed 3D numerical experiments, considering periodic boundary conditions in the spanwise direction while ignoring the side vortices to explore the influence of trailing edge vortices on the large-amplitude flapping of inverted flags. In a recent study, Goza et al. [11] employed an immersed boundary method to study the nonlinear response and stability of inverted flags and explored the effect of Reynolds number as well as the mass ratio parameter on the system dynamics for a wide range of flow velocities. They concluded that the mass ratio has a negligible effect on the onset of flapping. They also concluded that heavy flags are subject to a 'non-classical-VIV' flapping mechanism, in which, as opposed to 'classical VIV', the flapping regime is not the result of frequency

\footnotetext{
${ }^{1}$ In this paper, including the Introduction where others' work is also discussed, the mass ratio is as defined in the nomenclature (fluid/structure); in some other papers, the inverse definition (structure/fluid) is utilized.
} 
synchronisation between flapping and vortex shedding.

The analytical work by Sader et al. [24] and the numerical simulations by Goza et al. [11] showed the susceptibility of VIV to the mass ratio parameter. In contrast to light flags, the synchronisation of flapping and vortex shedding is lost in the case of heavy flags. Goza et al. [11] moreover demonstrated that at very low Reynolds numbers, where vortex shedding is essentially absent, large-amplitude flapping still occurs for heavy flags. Hence, they concluded that the mechanism generating the flapping motion of heavy flags is distinct from VIV. They attributed the flapping to the instability of the deformed equilibria of the system.

The non-VIV flapping motion identified by Goza et al. [11] has motivated the current study. The emergence of flapping motion for low mass ratio flags in low Reynolds number flow, where vortex shedding does not occur, impelled us to consider the importance of the effect of unsteady forces arising from changes in the fluid-flow momentum due to motion of the immersed flag. Of particular interest is the investigation of flow-induced oscillation of heavy inverted flags with mass ratios of $O(1)$, with the aid of well-established inviscid flow theories available in the literature. Indeed, the nonlinear large-amplitude flapping of an inverted flag is a challenging fully coupled fluid-structure interaction problem for which computational techniques are particularly expensive. Despite advances in CFD techniques and computing power, analytical models are still preferable for conducting simplified numerical simulations and interpreting experimental observations in a straightforward fashion. To further support the use of inviscid potential flow theory, it has been observed experimentally that the flow around the leading edge of the inverted flag reattaches at middle stroke, where the angle of attack is relatively large [36]. In addition, analytical inviscid expressions for the normal force acting on low-aspect-ratio plates have yielded good predictions for angles of attack up to approximately $40^{\circ}$, before massive separation at the leading edge and stall occurs $[2,33,5]$. Accordingly, inviscid forces are dominant, at least at the region with moderate angles of attack, thus justifying the preference for inviscid potential flow theory.

To the best of our knowledge, there is no analytical study in the literature on the nonlinear dynamical response of inverted flags subjected to axial flow accounting for velocity-dependent forces. The present paper aims at producing such a model in the limit case of slender flexible plates, and testing its capability to predict the dynamical behaviour of heavy slender inverted flags in axial flow, such as the onset of instabilities, frequency and amplitude of flapping, as well as elucidating the physical mechanisms involved in the transition between different dynamical states.

To this end, continuum representations of fluid-flow forces are detailed on the basis of the large amplitude elongated body theory [17] to formulate the reactive force and Bollay's model to express the nonlinear vortex lift mechanism, with no account for the vortex shedding mechanism from the leading edge. Hamilton's principle is employed to balance the kinetic and potential energies of the inverted flag and the virtual work of flow forces and damping. More specifically, the kinematics is covered in Section 2.1, constitutive equations in Section 2.2, and conditions of equilibrium leading to final equation of motion in Section 2.3. The resultant partial-integro-differential equation is discretized spatially by means of a conventional Galerkin procedure and is recast in a set of nonlinear ordinary differential equations (ODEs). The nonlinear response of the system is explored in detail by utilizing direct time-stepping integration together with bifurcation analysis. In particular, the ODEs are solved in time using Gear's backward differentiation, and bifurcation diagrams are constructed with the aid of the pseudo-arclength continuation technique. The sensitivity of the dynamics to the aspect ratio, mass ratio, incidence angle, and viscous drag is also explored. The results are summarized in the form of bifurcation diagrams to identify transitions between various regimes.

\section{Analytical model}

In this section, a nonlinear model is developed for the inverted flag/plate problem. First, the problem is defined mathematically. Expressions for fluid forces are formulated separately, and the nonlinear integro-partial-differential equations governing the rotation of the mid-plane are derived. These equations are non-dimensionalized and then discretized via the Galerkin method.

\subsection{Problem geometry}

The system considered is shown in Figure 1(a) consisting of a vertical cantilevered thin plate subjected to an inviscid axial flow impinging to its free end. The plate has length $L$, width $H$, thickness $h$, transverse moment of inertia $I$, cross-sectional area $A$, surface area $S$, and flexural rigidity $D$. The mechanical properties of the plate, i.e., its density, Poisson ratio, Young's modulus, and internal damping coefficient are denoted by $\rho_{\mathrm{p}}, \nu, E$, and $\eta$, respectively; $\rho_{\mathrm{f}}$ stands for the density of the fluid flowing with mean flow velocity $U$. A right-handed Cartesian reference system $(\mathrm{O} ; x, y, z)$ is considered, with axes $x \mathbf{i}$ and $z \mathbf{k}$ being the axial and the transverse directions, respectively. The transverse and longitudinal motions $w(x, t)$ and $u(x, t)$ of a generic point at a distance $z$ from the mid-plane on the cross-section of the flag are shown in Figure 1(b); $t$ denotes time. The inextensibility assumption [27] reads $\left(1+\partial_{x} u(x, t)\right)^{2}+\partial_{x} w(x, t)^{2}=1$, with $\partial_{x}$ denoting the spatial derivative; it enforces a constant flag length during deformation. As a consequence, $w$ and $u$ can be expressed in terms of only the slope $\psi$, which is also the rotation 
(a)

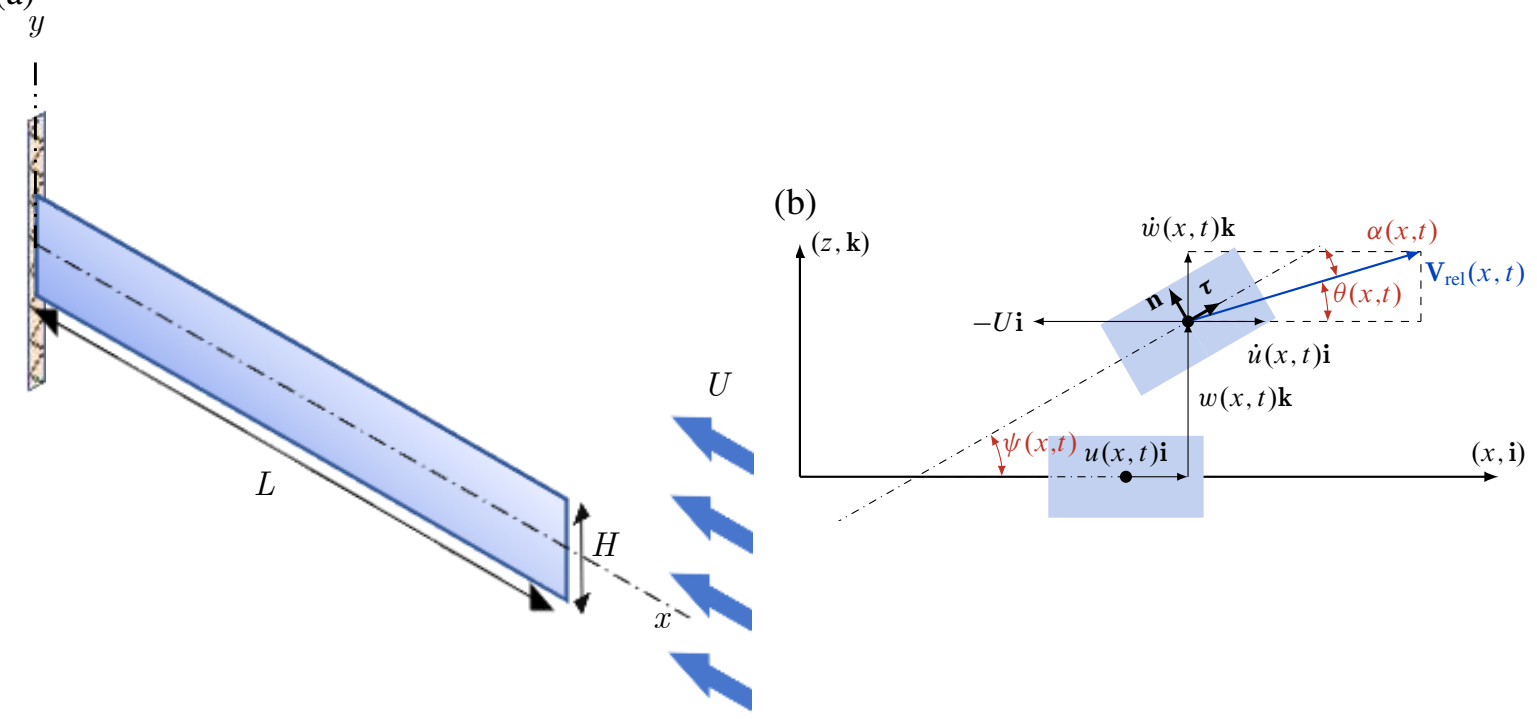

Figure 1: (a) Inverted flag, idealized as a thin Euler-Bernoulli beam in axial flow, free at the upstream end and clamped at the downstream end, (b) Generic infinitesimal element of the beam.

angle of the cross-section. Given that $\partial_{x} w(x, t)=\cos \psi(x, t)$ and $1+\partial_{x} u(x, t)=\sin (x, t)$, the corresponding velocities are formulated as

$$
\begin{aligned}
& \dot{w}(x, t)=\int_{0}^{x} \partial_{s} \dot{w}(s, t) \mathrm{d} s=\int_{0}^{x} \dot{\psi}(s, t) \cos \psi(s, t) \mathrm{d} s \\
& \dot{u}(x, t)=\int_{0}^{x} \partial_{s} \dot{u}(s, t) \mathrm{d} s=-\int_{0}^{x} \dot{\psi}(s, t) \sin \psi(s, t) \mathrm{d} s .
\end{aligned}
$$

Considering a deflected plate, the relative velocity of the solid with respect to the incident flow reads $\mathbf{V}_{\text {rel }}(x, t)=$ $(\dot{u}(x, t)+U) \mathbf{i}+\dot{w}(x, t) \mathbf{k}$. Upon projection on the normal and tangential directions, the normal and the tangential components of the relative velocity respectively are:

$$
\begin{aligned}
\mathbf{V}_{\mathrm{n}}(x, t) & =(-(\dot{u}+U) \sin \psi+\dot{w} \cos \psi) \mathbf{n}, \\
\mathbf{V}_{\tau}(x, t) & =((\dot{u}+U) \cos \psi+\dot{w} \sin \psi) \boldsymbol{\tau} .
\end{aligned}
$$

Defining the instantaneous angle of attack $\alpha$ as the instantaneous inclination between the chord-line and the relative velocity vector, one obtains

$$
\alpha=\psi-\operatorname{atan}\left(\frac{\dot{w}}{U+\dot{u}}\right)
$$

\subsection{Fluid modelling}

The purpose of the present study is to gain insight into the factors affecting the dynamics of small aspect ratio inverted flags. While this could be treated by fully-coupled fluid-structure interaction (FSI) solvers and CFD schemes, it is of interest to develop a model which is predominantly analytical. This provides an opportunity to better understand the underlying aerodynamic effects. Besides, direct numerical simulation of such a complex FSI problem is particularly expensive. Hence, an analytical expression of the fluid flow forces is developed in the present study. The flow is analyzed as if it were inviscid in order to find the pressure-related forces, provided that there is no flow separation at the leading edge, even for relatively large angles of attack. The strong vortex formation along the lateral edges of the flag is approximated by Bollay's nonlinear wing theory [2]. Then, semi-analytical expressions are added for viscous forces acting on the plate. It is nevertheless recognized that the applicability of inviscid theory depends on whether the flow remains attached. Hence, the predictions for aspect ratios larger than unity, for which massive separation occurs, are limited to small angles of attack [5].

The proposed model is based on the large amplitude elongated body theory [17] to obtain the reactive fluid forces associated with the relative motion of the inverted flag with respect to the incident flow. This model has been successfully employed in a number of studies, showing the reliability of predictions of the flapping motion in comparison with Navier-Stokes simulations [3] and a full three-dimensional panel method [37] for aspect ratios of $O(1)[6,34,8]$. According to the large amplitude elongated body theory, the reactive force relates to the balance of 
the rate of change of solid and fluid momenta and the pressure force acting in the tangential direction corresponding to the quadratic terms in Bernoulli's equation [16]. As such, the inviscid pressure force is expressed as

$$
F_{\mathrm{N}}=\frac{\pi}{4} \rho_{\mathrm{f}} H^{2}\left[-\partial_{t}\left(V_{\mathrm{n}} \mathbf{n}\right)+\partial_{x}\left(V_{\mathrm{n}} V_{\tau} \mathbf{n}-\frac{1}{2} V_{n}^{2} \boldsymbol{\tau}\right)\right]
$$

The $\mathbf{n}$ component of $F_{\mathrm{N}}$ gives the magnitude of the reactive force (per unit length) acting on the flag, as shown in Figure 2. Inserting the expressions for the normal and tangential components of the relative velocity in Eq. (4) gives

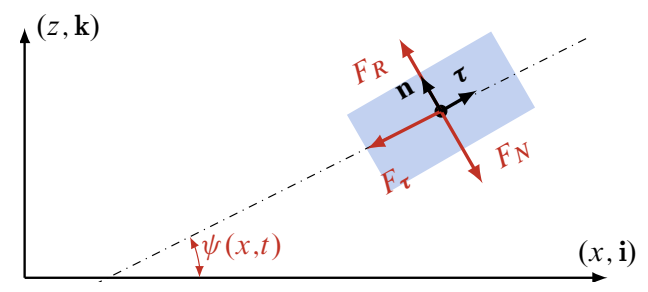

Figure 2: Schematic of the forces acting on a generic infinitesimal element of the plate.

$$
\begin{aligned}
F_{\mathrm{N}}= & \frac{\pi}{4} \rho_{\mathrm{f}} H^{2}\left[\ddot{w} \cos \psi-\ddot{u} \sin \psi+2 \dot{\psi}(\dot{u} \cos \psi+\dot{w} \sin \psi)+\frac{3}{2} \partial_{x} \psi\left(\dot{w}^{2}-\dot{u}^{2}\right) \cos ^{2} \psi\right. \\
& +\partial_{x} \psi\left(\dot{u}^{2} / 2-3 \dot{u} \dot{w} \cos \psi \sin \psi-\dot{w}^{2}\right)+U\left(\left(\dot{u}-3 \dot{u} \cos ^{2} \psi-3 \dot{w} \sin \psi \cos \psi\right) \partial_{x} \psi+2 \dot{\psi} \cos \psi\right) \\
& \left.-\frac{1}{2} U^{2}\left(3 \cos ^{2} \psi-1\right) \partial_{x} \psi\right] .
\end{aligned}
$$

The nonlinear vortex lift associated to the circulation of the side-edge vortices and the distribution of spanwise vorticity is devised based on Bollay's nonlinear wing theory [2]. Briefly, Bollay obtained the induced velocity originating from the system of vortices at a generic point of the plate and calculated the normal force per unit length associated with the cross-flow component of the flow velocity. The expression for the normal force given in [2] is not explicit, though approximate expressions can be sought based on the assumption made by Gersten in [10] $]^{2}$ or analogously based on Polhamus' work [23,31]. Hence, the normal force reads

$$
F_{\mathrm{R}}=\frac{\rho_{\mathrm{f}} H U^{2}}{2}\left(K_{\mathrm{p}}(R) \sin \alpha \cos \alpha+K_{\mathrm{v}}(R) \sin ^{2} \alpha\right)
$$

where $K_{\mathrm{p}}$ and $K_{\mathrm{v}}$ are coefficients reflecting the circulation and side-edge vortex flow, and are determined for various aspect ratios, according to the technique implemented in [31]; $R$ denotes the aspect ratio of the flag. The curves obtained for the $K_{\mathrm{p}}$ and $K_{\mathrm{v}}$ coefficients over a range of aspect ratios is shown in Figure 3; the reader is referred to a previous study [31] by the authors for more details. These values depend only on the aspect ratio of the inverted

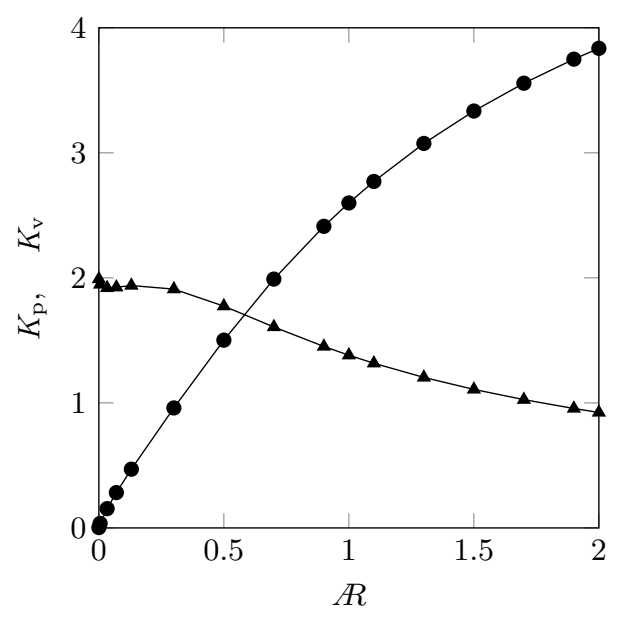

Figure 3: Variation of $K_{\mathrm{p}}[\bullet]$ and $K_{\mathrm{v}}[\mathbf{\Delta}]$ with the aspect ratio of the flag, $A$.

flag. For instance, for the case of an infinitely long plate (i.e., zero aspect ratio), $K_{\mathrm{v}}=2$ and $K_{\mathrm{p}}=0$. The value of

\footnotetext{
${ }^{2}$ Gersten assumed that the flow trails off the surface of the plate at an angle equal to half of the plate's angle of attack. Hence, the expression for the normal force takes on an explicit form in terms of $\alpha$.
} 
$K_{\mathrm{v}}\left(K_{\mathrm{p}}\right)$ decreases (increases) with an increase in the flag aspect ratio and approaches zero $(2 \pi)$ as the aspect ratio approaches infinity. Inserting Eq. (3) in Eq. (6), one obtains

$$
\begin{aligned}
F_{\mathrm{R}}=\frac{\rho_{\mathrm{f}} H U^{2}}{2(\dot{u}+U)^{2}+2 \dot{w}^{2}} & {\left[K_{\mathrm{p}}\left[\left((\dot{u}+U)^{2}-\dot{w}^{2}\right) \sin \psi \cos \psi-(\dot{w} \dot{u}+U \dot{w}) \cos 2 \psi\right]\right.} \\
+ & \left.K_{\mathrm{v}}\left[(\dot{u}+U)^{2} \sin ^{2} \psi+\dot{w}^{2} \cos ^{2} \psi-2(\dot{w} \dot{u}+U \dot{w}) \sin \psi \cos \psi\right]\right] .
\end{aligned}
$$

The uniform distribution of tangential aerodynamic force acting on the flag due to the viscous effects of the fluid flow simplifies to

$$
F_{\tau}=\frac{1}{2} \rho_{\mathrm{f}} H U^{2} K_{\tau}(R e) \cos \alpha
$$

where $K_{\tau}$ is the coefficient of the effective drag caused by viscous stresses acting on the flag and can be determined using the semi-empirical expressions proposed by Taylor [32] (see, for instance [26]) or utilizing a Blasius-type solution for either turbulent or laminar flows (see, for instance [34]). This allows for the approximate treatment of the viscous drag. The effect of $K_{\tau}$ on the system dynamics is discussed through extensive numerical tests in Section 3.6.

\subsection{Equation of motion}

The inverted flag is idealized as a cantilevered elastic beam shown in Figure 1. The conventional nonlinear EulerBernoulli beam theory is considered and the equation of motion is obtained via Hamilton's principle. The variation of the kinetic energy of the inverted flag system is given by

$$
\int_{t_{1}}^{t_{2}} \delta \mathcal{T}(t) \mathrm{d} t=\int_{t_{1}}^{t_{2}}\left[\rho_{\mathrm{p}} A \int_{0}^{L}(\dot{w} \delta \dot{w}+\dot{u} \delta \dot{u}) \mathrm{d} x+\rho_{\mathrm{p}} I \int_{0}^{L} \dot{\psi} \delta \dot{\psi} \mathrm{d} x\right] \mathrm{d} t
$$

$I=H h^{3} / 12$ being the second moment of inertia. The expression for the variation of the strain energy of the system is expressed as

$$
\int_{t_{1}}^{t_{2}} \delta \mathcal{V}(t) \mathrm{d} t=\int_{t_{1}}^{t_{2}} D \int_{0}^{L} \partial_{x} \psi(x, t) \partial_{x} \delta \psi(x, t) \mathrm{d} x \mathrm{~d} t,
$$

where $D=E I$ denotes the flexural rigidity of a slender inverted flag; for a flag of large aspect ratio, the plane-strain flexural rigidity $D=E I /\left(1-v^{2}\right)$ is considered. The virtual work associated with the fluid-dynamic forces can be expressed as

$$
\delta W_{\mathrm{f}}(t)=\int_{0}^{L}\left[-\left(\left(F_{\mathrm{N}}+F_{\mathrm{R}}\right) \cos \psi-F_{\tau}(x, t) \sin \psi\right) \delta w+\left(\left(F_{\mathrm{N}}-F_{\mathrm{R}}\right) \sin \psi-F_{\tau} \cos \psi\right) \delta u\right] \mathrm{d} x .
$$

The virtual work done by the Kelvin-Voigt structural damping and the dissipation due to fluid viscosity is

$$
\delta W_{\mathrm{d}}(t)=-D \eta \int_{0}^{L} \partial_{x x} \dot{\psi} \delta \psi \mathrm{d} x-c \int_{0}^{L}\left(\partial_{t}\left[\int_{0}^{x} \cos \psi(\xi, t) \mathrm{d} s\right] \delta u+\partial_{t}\left[\int_{0}^{x} \sin \psi(\xi, t) \mathrm{d} s\right] \delta w\right) \mathrm{d} x
$$

where $\eta$ is the material viscosity damping and $c$ the fluid damping coefficient $[8]^{3}$. Inserting expressions (8), (7) and (5) into Eq. (11) and substituting the resultant along with Eqs. (9), (10) and (12) into the expression for the extended Hamilton's principle

$$
\int_{t_{1}}^{t_{2}}\left(\delta \mathcal{T}(t)-\delta \mathcal{V}(t)+\delta W_{\mathrm{f}}(t)+\delta W_{\mathrm{d}}(t)\right) \mathrm{d} t=0
$$

yields

$$
\begin{aligned}
\rho_{\mathrm{p}} I \partial_{t t} \psi-\rho_{\mathrm{p}} A \sin \psi \partial_{t t}\left(\int_{x}^{L} \int_{0}^{s} \cos \psi(\xi, t) \mathrm{d} \xi \mathrm{d} s\right)+\rho_{\mathrm{p}} A \cos \psi \partial_{t t}\left(\int_{x}^{L} \int_{0}^{s} \sin \psi(\xi, t) \mathrm{d} \xi \mathrm{d} s\right)-D \eta \partial_{t x x} \psi \\
-D \partial_{x x} \psi+\cos \psi \int_{L}^{x}\left(\left(F_{\mathrm{N}}(s, t)-F_{\mathrm{R}}(s, t)\right) \cos \psi(s, t)-F_{\tau}(s, t) \sin \psi(s, t)\right) \mathrm{d} s \\
+c\left[\cos \psi \partial_{t}\left(\int_{x}^{1} \int_{0}^{s} \sin \psi(\xi, t) \mathrm{d} \xi \mathrm{d} s\right)-\sin \psi \partial_{t}\left(\int_{x}^{1} \int_{0}^{s} \cos \psi(\xi, t) \mathrm{d} \xi \mathrm{d} s\right)\right] \\
\quad+\sin \psi \int_{L}^{x}\left(\left(F_{\mathrm{N}}(s, t)-F_{\mathrm{R}}(s, t)\right) \sin \psi(s, t)+F_{\tau}(s, t) \cos \psi(s, t)\right) \mathrm{d} s=0
\end{aligned}
$$

\footnotetext{
${ }^{3}$ This model of damping in quiescent fluid is an approximation taking into account the dissipative effect due to the surrounding fluid. It has been employed in work involving cylinders oscillating in fluid [22, 1]. A more comprehensive approach to model the viscous forces is through a full viscous flow calculation using computational techniques, a very challenging task beyond the purposes of this paper.
} 
Utilizing the dimensionless parameters $x^{*}=x / L, t^{*}=t / \tau, \beta=I /\left(A L^{2}\right), k_{\tau}=K_{\tau} / 2, k_{\mathrm{p}}=K_{\mathrm{p}} / 2$, $k_{\mathrm{v}}=K_{\mathrm{v}} / 2, \eta_{\mathrm{s}}=\eta / \tau, f^{*}=f \tau, \mu=\rho_{\mathrm{f}} L /\left(\rho_{\mathrm{p}} h\right), R=H / L, \Pi=\rho_{\mathrm{f}} S L^{2} U^{2} / D$, and $c_{\mathrm{d}}^{*}=c_{\mathrm{d}} L^{4} /(D \tau)$, in which $f$ is the frequency of flapping and $\tau=\sqrt{\rho_{\mathrm{p}} A / D} L^{2}$, yields the following dimensionless nonlinear equation of motion (in which the asterisk notation has been dropped for simplicity):

$$
\begin{gathered}
\beta \ddot{\psi}-\sin \psi \partial_{t t}\left(\int_{x}^{1} \int_{0}^{s} \cos \psi(\xi, t) \mathrm{d} \xi \mathrm{d} s\right)+\cos \psi \partial_{t t}\left(\int_{x}^{1} \int_{0}^{s} \sin \psi(\xi, t) \mathrm{d} \xi \mathrm{d} s\right) \\
-\partial_{x x} \psi+c_{\mathrm{d}}\left[\cos \psi \partial_{t}\left(\int_{x}^{1} \int_{0}^{s} \sin \psi(\xi, t) \mathrm{d} \xi \mathrm{d} s\right)-\sin \psi \partial_{t}\left(\int_{x}^{1} \int_{0}^{s} \cos \psi(\xi, t) \mathrm{d} \xi \mathrm{d} s\right)\right] \\
-\eta_{\mathrm{s}} \partial_{t x x} \psi+\Pi\left[\cos \psi \int_{1}^{x}\left(C_{\mathrm{f}}(s, t) \cos \psi(s, t)-C_{\tau}(s, t) \sin \psi(s, t)\right) \mathrm{d} s\right. \\
\left.+\sin \psi \int_{1}^{x}\left(C_{\mathrm{f}}(s, t) \sin \psi(s, t)+C_{\tau}(s, t) \cos \psi(s, t)\right) \mathrm{d} s\right]=0 .
\end{gathered}
$$

Using the notation $\gamma=\sqrt{\mu / \Pi}$,

$$
\begin{aligned}
C_{\mathrm{f}}=\frac{\pi R}{4} \gamma[\gamma(\ddot{w} \cos \psi-\ddot{u} \sin \psi+2 \dot{\psi}(\dot{u} \cos \psi+\dot{w} \sin \psi) \\
\left.\quad+\partial_{x} \psi\left(3 \cos ^{2} \psi\left(\dot{w}^{2}-\dot{u}^{2}-1\right)+1+\dot{u}^{2}-6 \dot{u} \dot{w} \cos \psi \sin \psi-2 \dot{w}^{2}\right) / 2\right) \\
\left.\quad+\partial_{x} \psi\left(\dot{u}-3 \dot{u} \cos ^{2} \psi-3 \dot{w} \sin \psi \cos \psi\right)+2 \dot{\psi} \cos \psi\right] \\
-\frac{k_{\mathrm{p}}(R)}{\mathrm{B}^{2}}\left[\left((\gamma \dot{u}+1)^{2}-\gamma \dot{w}^{2}\right) \sin \psi \cos \psi+\gamma(\gamma \dot{w} \dot{u}+\dot{w})\left(\sin ^{2} \psi-\cos ^{2} \psi\right)\right] \\
-\frac{k_{\mathrm{v}}(R)}{\mathrm{B}^{2}}\left[(\gamma \dot{u}+1) \sin ^{2} \psi+\gamma^{2} \dot{w}^{2} \cos ^{2} \psi-2 \gamma(\gamma \dot{w} \dot{u}+\dot{w}) \sin \psi \cos \psi\right]
\end{aligned}
$$

and

$$
C_{\tau}=\frac{k_{\tau}(\operatorname{Re})}{\mathrm{B}}[(1+\gamma \dot{u}) \cos \psi+\gamma \dot{w} \sin \psi]
$$

in which $\mathrm{B}=\sqrt{(1+\gamma \dot{u})^{2}+\gamma^{2} \dot{w}^{2}}$.

Also, it is recalled that the boundary conditions for the clamped-free flag read $\psi(0, t)=0$ and $\psi_{x}(L, t)=0$.

The discretization of the system in space is obtained via the Galerkin technique. Hence, as a comparison functions, a family of eigenfunctions $\phi_{r}, r=1, \ldots, M$, satisfying the geometric boundary conditions of the problem, is selected to approximate the deflection pattern of the plate [21, Section 2.1.4]. The rotation angle $\psi(x, t)$ is then expanded as

$$
\psi(x, t)=\sum_{r=1}^{M} \phi_{r}(x) q_{r}(t) \text { with } \phi_{r}(x)=\sin \left(\lambda_{r} x\right)+\sinh \left(\lambda_{r} x\right)+\frac{\cos \left(\lambda_{r}\right)+\cosh \left(\lambda_{r}\right)}{\sin \left(\lambda_{r}\right)+\sinh \left(\lambda_{r}\right)}\left(\cos \left(\lambda_{r} x\right)-\cosh \left(\lambda_{r} x\right)\right)
$$

where $q_{r}(t)$ are the unknown time-dependent generalized coordinates and $M$ is the number of modes used in the analysis. Plugging Eq. (16) into Eq. (1) and Eq. (3), inserting the resultant in Eq. (15), and applying Galerkin's technique, yields a set of $M$ second-order ODEs with trigonometric nonlinearities.

\section{System dynamics}

The set of ODEs is solved by means of the AUTO software [7, 18] which uses the pseudo-arclength continuation technique to construct bifurcation diagrams. This technique performs continuation of both stable and unstable solution branches. Time integration is performed via Gear's backward differentiation formula [9]. For the problem at hand, the DIVPAG routine of the IMSL library is used. Time histories of the amplitude of oscillation are used to plot phase-plane portraits, Fast Fourier transforms (FFT), and static as well as flapping flag shapes at points of interest in the parameter space. The FFT is obtained once the transient response has decayed, and the mean amplitude of each harmonic is calculated. Flag shapes are retrieved through Eq. (1). In all bifurcation diagrams, continuous and dashed lines represent stable and unstable branches of the solution, respectively. Each colour used therein corresponds to a specific regime in the range of flow velocities considered.

\subsection{Numerical parameters}

In this study, six modes are employed to discretize Eq. (15) (i.e. $M=6$ ); this is sufficient to reach convergence. Moreover, the following parameters are utilized [25]: $L=30 \mathrm{~cm}, h=1 \mathrm{~mm}, \rho_{\mathrm{p}}=1200 \mathrm{~kg} \mathrm{~m}^{-3}, \rho_{\mathrm{f}}=1.2 \mathrm{~kg} \mathrm{~m}^{-3}$, $E=2.4 \mathrm{GPa}, v=0.38$, and $\eta_{\mathrm{s}}=0.008$. The viscous damping coefficient $c_{\mathrm{d}}$ is replaced by $2 \zeta \omega_{1}$ where $\zeta$ is the modal damping ratio and $\omega_{1}$ is the first dimensionless natural frequency. The modal damping ratio $\zeta$ is set at 0.05 . The dynamics of the system depends on five dimensionless parameters: the dimensionless flow velocity $\Pi$, the flag aspect ratio $R$, the incidence angle $\psi_{0}$, and the viscous drag coefficient $k_{\tau}$. Here, $\Pi$ is selected as the bifurcation parameter while other parameters are kept constant. 


\subsection{Possible regimes}

With the dimensionless parameters $\mu=0.3, R=0.5$ and $k_{\tau}=0.01$ which yield $k_{\mathrm{p}}=0.745$ and $k_{\mathrm{v}}=0.895$, four distinct regimes are captured; the corresponding bifurcation diagram is displayed in Figure 4 . As the flow
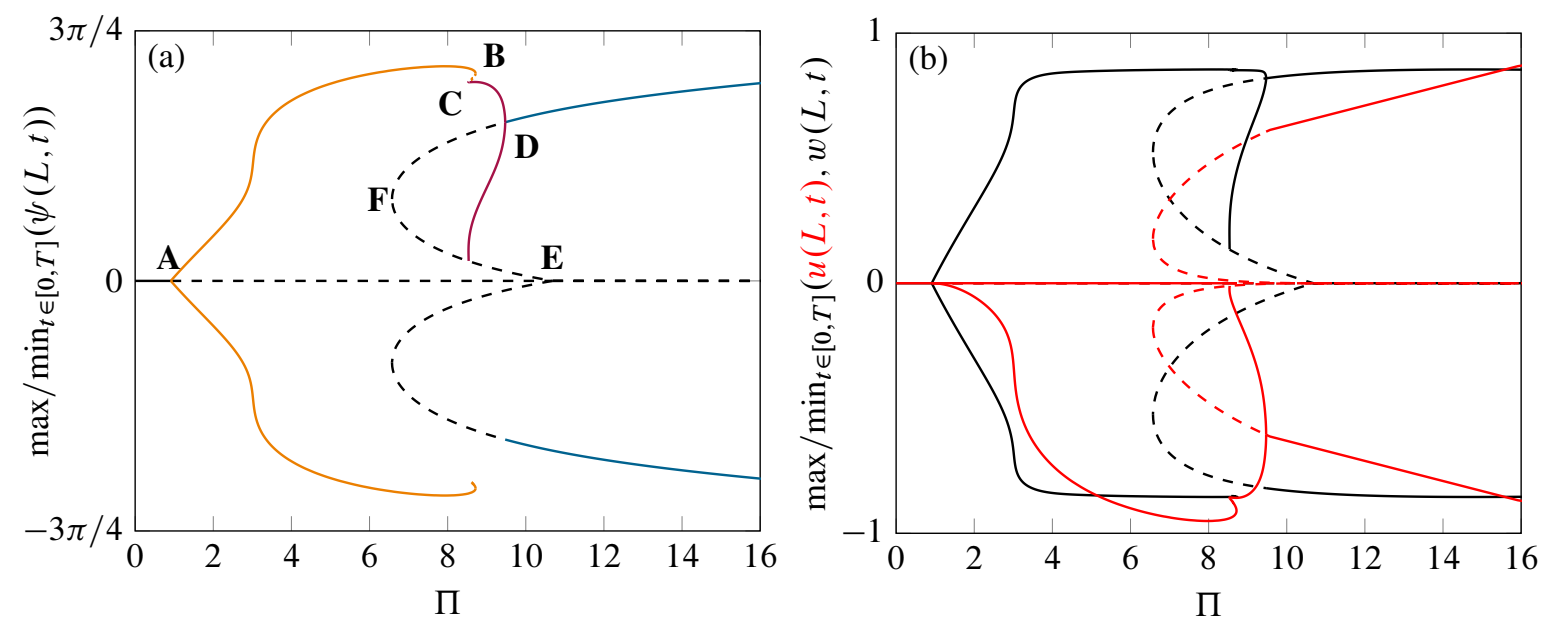

Figure 4: Bifurcation diagrams for $\mu=0.3$ and $R=0.5$. (a) Maximum/minimum values of the tip slope of the inverted flag over a cycle of steady-state oscillation of period T. [-] Stable static solution (undeflected stable equilibrium), [-..] unstable static solution, [-] stable periodic solution (oscillation around undeflected equilibrium), [-] stable periodic solution (oscillations around deflected equilibria), and [-] stable static solution (deflected static equilibria). (b) maximum/minimum values of the tip transverse(black)/longitudinal(red) motion over a cycle of steady-state oscillation of period $T$. The colour scheme in (a) is used in Figs. 5-8.

velocity is increased, a sequence of regimes emerges as follows:

i) The undeflected static equilibrium where the inverted flag is stable. Small disturbances generate a motion that ultimately dies out.

ii) The undeflected static equilibrium loses stability at point $\mathrm{A}$ where $\Pi=0.91$ via a supercritical Hopf bifurcation from which emanates limit-cycle motion ${ }^{4}$. In other words, the inverted flag then undergoes a large-amplitude flapping motion around the undeflected static equilibrium. This regime survives over a range of $\Pi \in[0.918 .71]$ and the amplitude of flapping increases with flow velocity. Figure 5 shows the time history, phase-plane portrait and FFT-plot harmonic content with the frequency of oscillation $f=0.40$ at $\Pi=6.00$, from which a periodic flapping motion is identified which develops after the transient regime.
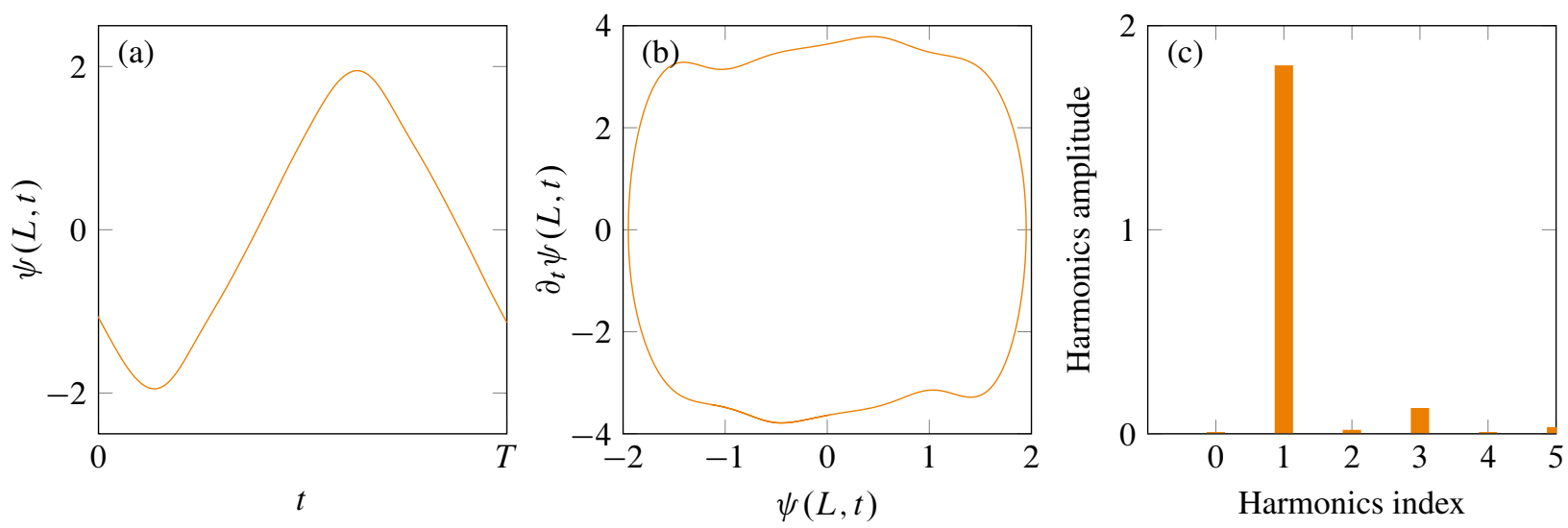

Figure 5: Periodic response of the inverted flag with $\mu=0.3$ and $R=0.5$ at $\Pi=6.0$ displaying a periodic motion around the trivial equilibrium; (a) time history, (b) phase-plane portrait, (c) and FFT plot indicating the frequency content of the response. Colour scheme from Figure 4(a).

iii) Increasing the flow velocity causes a transition from the flapping regime around the undeflected equilibrium to a deformed-flapping regime wherein the oscillation is around a deflected equilibrium. Transition between

\footnotetext{
${ }^{4}$ If the limit cycle is unstable or repelling, the Hopf bifurcation is of subcritical type; otherwise, the bifurcation is supercritical [21, Section 2.3].
} 
these regimes is accompanied by a jump in the amplitude and frequency of flapping via two saddle-node bifurcations at points $\mathrm{B}$ and $\mathrm{C}$ where $\Pi=8.71$ and $\Pi=8.53$, respectively. This motion can be observed in the range $\Pi \in[8.53$ 9.47]. Figure 6 depicts the time history and the phase-plane portrait at $\Pi=9.00$ displaying a periodic motion, as well as a FFT plot showing the harmonic content of the response; the frequency of oscillation is $f=0.55$.
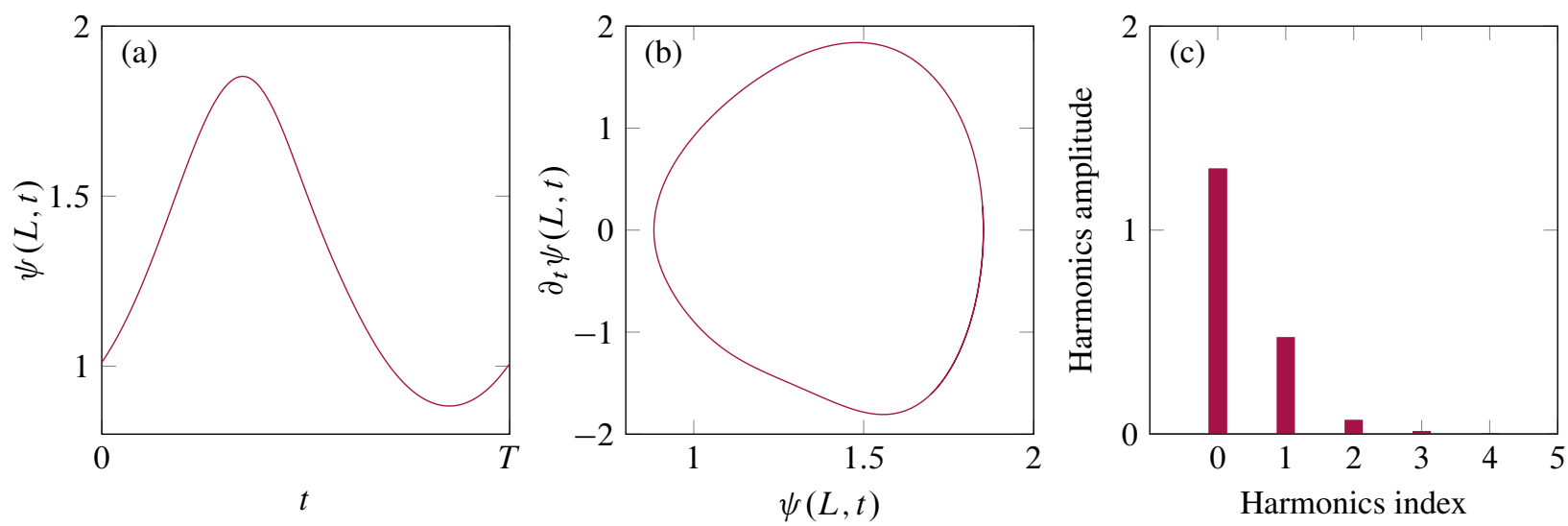

Figure 6: Periodic response of an inverted flag with $\mu=0.3$ and $R=0.5$ at $\Pi=9.0$ displaying a periodic motion around the nontrivial equilibrium; (a) time history, (b) phase-plane portrait, and (c) and FFT plot indicating the frequency content of the response. Colour scheme from Figure 4(a).

iv) The flapping motion ceases upon further increase in the flow velocity. The system response switches to a fully-deflected regime via a Hopf bifurcation at point $D$ where $\Pi=9.47$. Beyond that, the response of the system is attracted to stable large-amplitude deflected equilibria, and the inverted flag bends over one side. The tip rotation increases with flow velocity and the tip transverse deflection remains almost constant at its maximum value.

It should be noted that the response of the system is symmetric with respect to the undeflected equilibrium state and the inverted flag may flap around deflected equilibria (buckled state) on either side. In this paper, flapping around one side is plotted only, so that one can properly distinguish flapping around the undeflected static equilibrium and flapping around the deflected static equilibria. Both trivial and nontrivial unstable equilibria of the system, connecting the two Hopf bifurcation points, are shown in the bifurcation diagram as dashed lines. It is seen that, for the system studied here, the nontrivial static solution emerges from the bifurcation point $\mathrm{E}$ where $\Pi=10.6$ and folds at point $\mathrm{F}$ where $\Pi=6.6$.

The variation of the dimensionless frequency with $\Pi$ is plotted in Figure 7. With increasing $\Pi$, the flapping frequency decreases first and then recovers slightly at $\Pi=2.95$. The frequency increases sharply and monotonically for $\Pi \in[8.53$ 9.47], wherein the flapping around the deflected equilibrium takes place. This change in trend is accompanied by an abrupt increase in frequency.

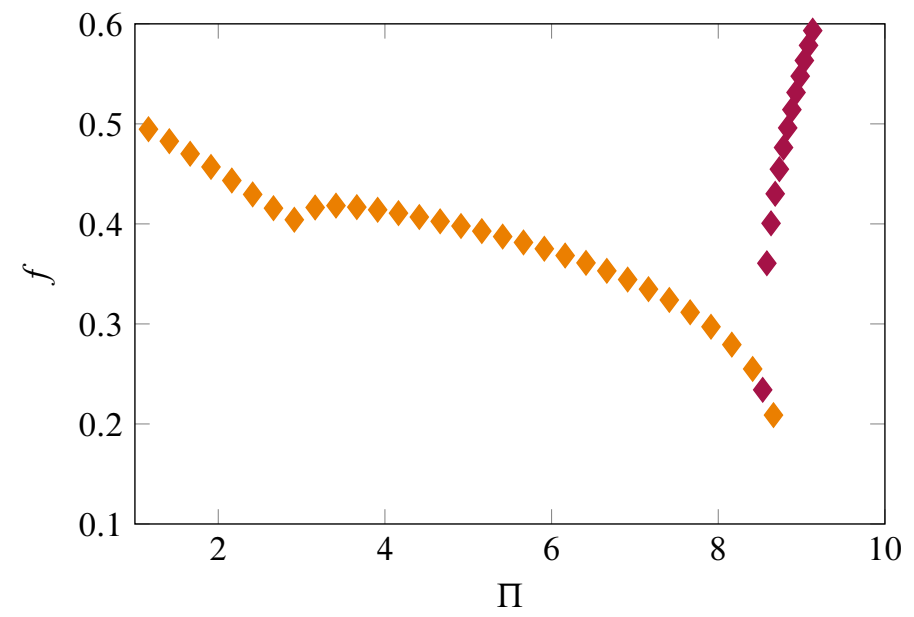

Figure 7: Dimensionless frequency $f$ versus dimensionless flow velocity $\Pi$ for an inverted flag with $\mu=0.3$ and $R=0.5$. Colour scheme from Figure 4(a).

In order to compare the response of the system in different regimes further, the (flapping) shapes of the inverted flag system are plotted in Figure 8. Subfigure 8(a) shows the undeflected flag for velocities below the critical value, 

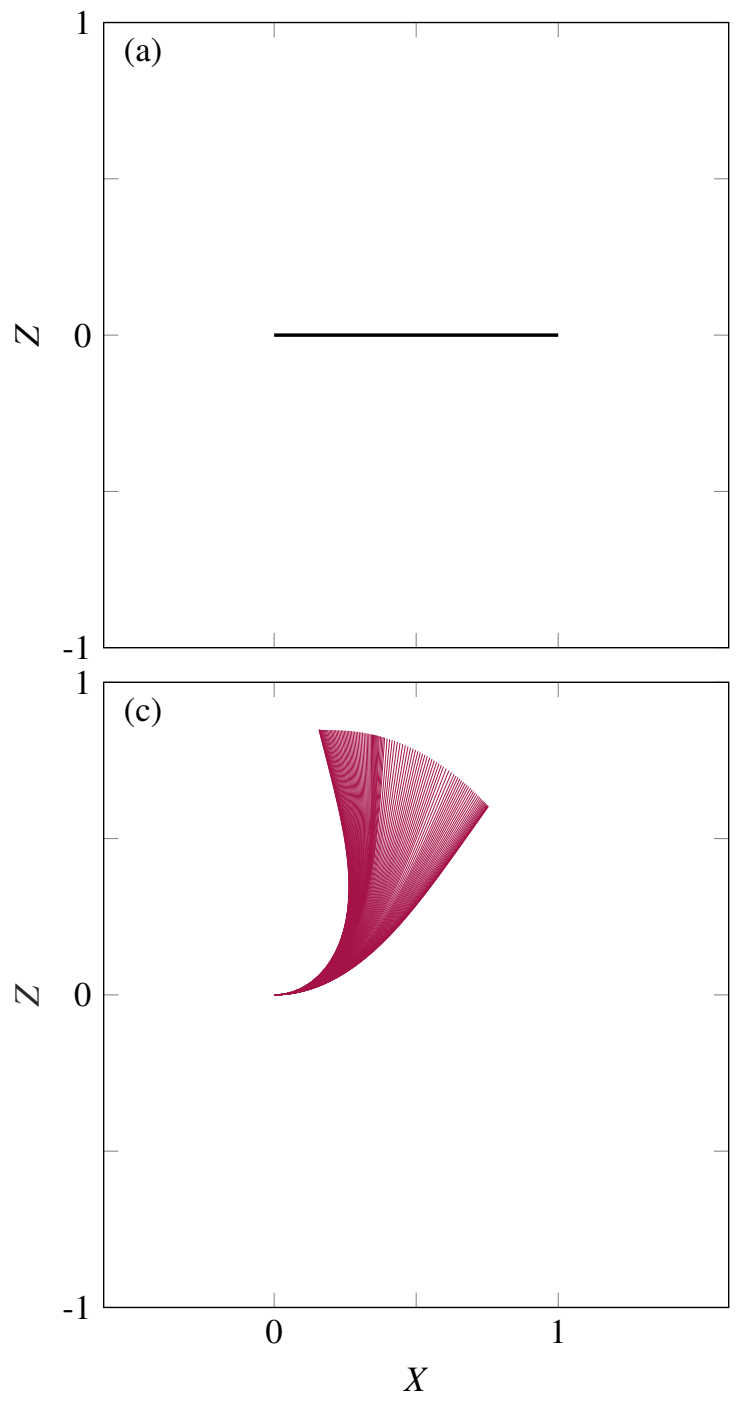
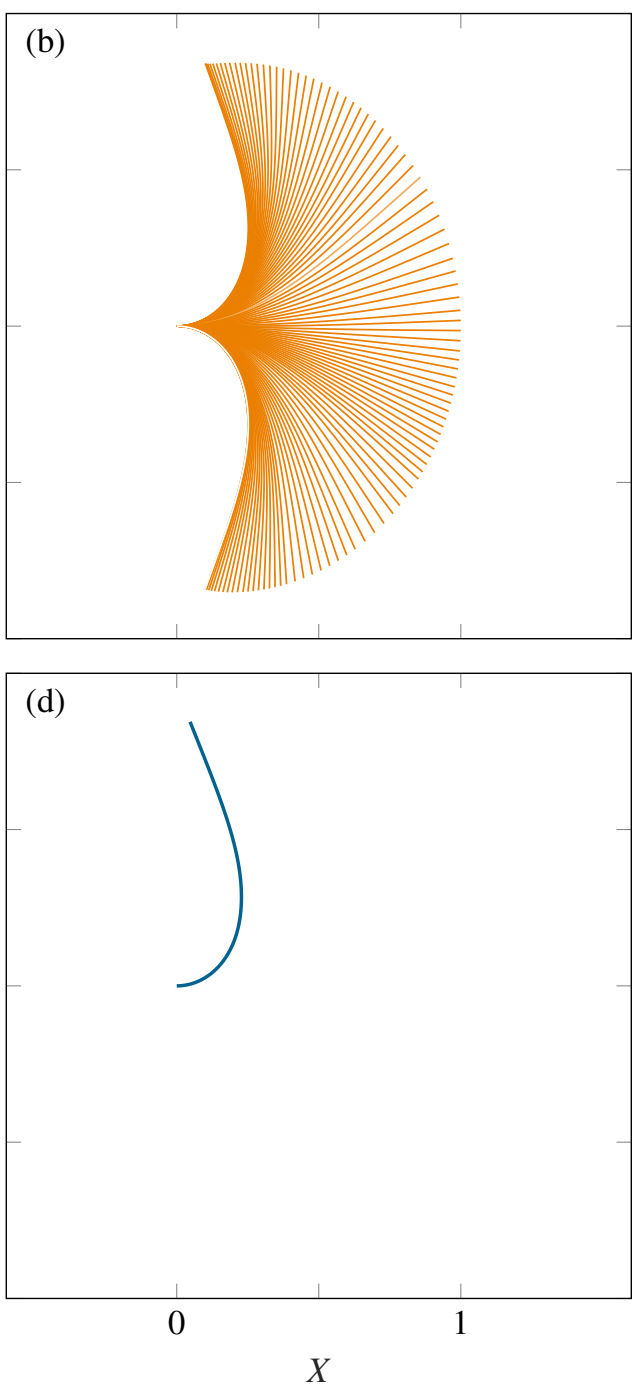

Figure 8: Dynamics obtained by superimposed deformations of an inverted flag with $\mu=0.3$ and $R=0.5$ at different flow velocities displaying (a) straight (undeflected) static equilibrium at $\Pi=0.80$, (b) large-amplitude flapping around the undeflected equilibrium at $\Pi=6.00$, (c) flapping around the deflected equilibrium at $\Pi=9.00$, and (d) fully-deflected state at $\Pi=20.00$. The colour scheme is defined in Figure 4(a).

that is $\Pi \leq 0$.91. In subfigure 8 (b), the inverted flag displays large-amplitude flapping motion at $\Pi=6.00$. It can be noticed that the transverse motion of the system is predominantly in the fundamental mode of a cantilevered beam. The deformed-flapping regime (oscillation around the deflected equilibrium) can be observed in subfigure 8(c) where $\Pi=9.00$, and finally, the fully-deflected state is shown in subfigure $8(d)$ for $\Pi=20.00$. It is nevertheless recognized that the validity of the proposed model deteriorates when the flag exhibits highly curved shapes. Indeed, at very large angles of attack, massive separation occurs leading to the normal force stall, which in turn can cause a sudden decrease in the amplitude of oscillation. The normal force stall phenomenon cannot be captured by inviscid flow theory, which naturally, neglects such viscous effects. Furthermore, the model ignores the effects of the unsteady vortex shedding at high flow rate in the fully-deflected regime; the results shown for this regime should be regarded with some caution, considering this limitation.

\subsection{Sensitivity to aspect ratio}

It is clear from Eqs. (5) and (7) that the coefficients of fluid forces are functions of the aspect ratio. Accordingly, the contribution of the fluid forces to the system dynamics varies with the aspect ratio. In order to investigate the effect of the aspect ratio on the response, four different cases are considered: flags of $H=3 \mathrm{~cm}, H=7.5 \mathrm{~cm}$, $H=10.5 \mathrm{~cm}$ and $H=15 \mathrm{~cm}$, associated with $R=0.1, R=0.25, R=0.35$ and $R=0.5$, respectively; other parameters are kept constant. The corresponding bifurcation diagrams are plotted in Figure 9. As seen in subfigure 9(a), flapping motions do not exist for $R=0.1$. When the flow velocity increases, the inverted flag deflects abruptly from its stable undeflected equilibrium to a stable deflected equilibrium via a subcritical pitchfork bifurcation at $\Pi=33.1$. Thus, the inverted flag may undergo a sudden large-amplitude deformation under small perturbation, even at flow velocities lower than the critical value (i.e. $\Pi \leq 33.1$ ) due to the emergence of stable 

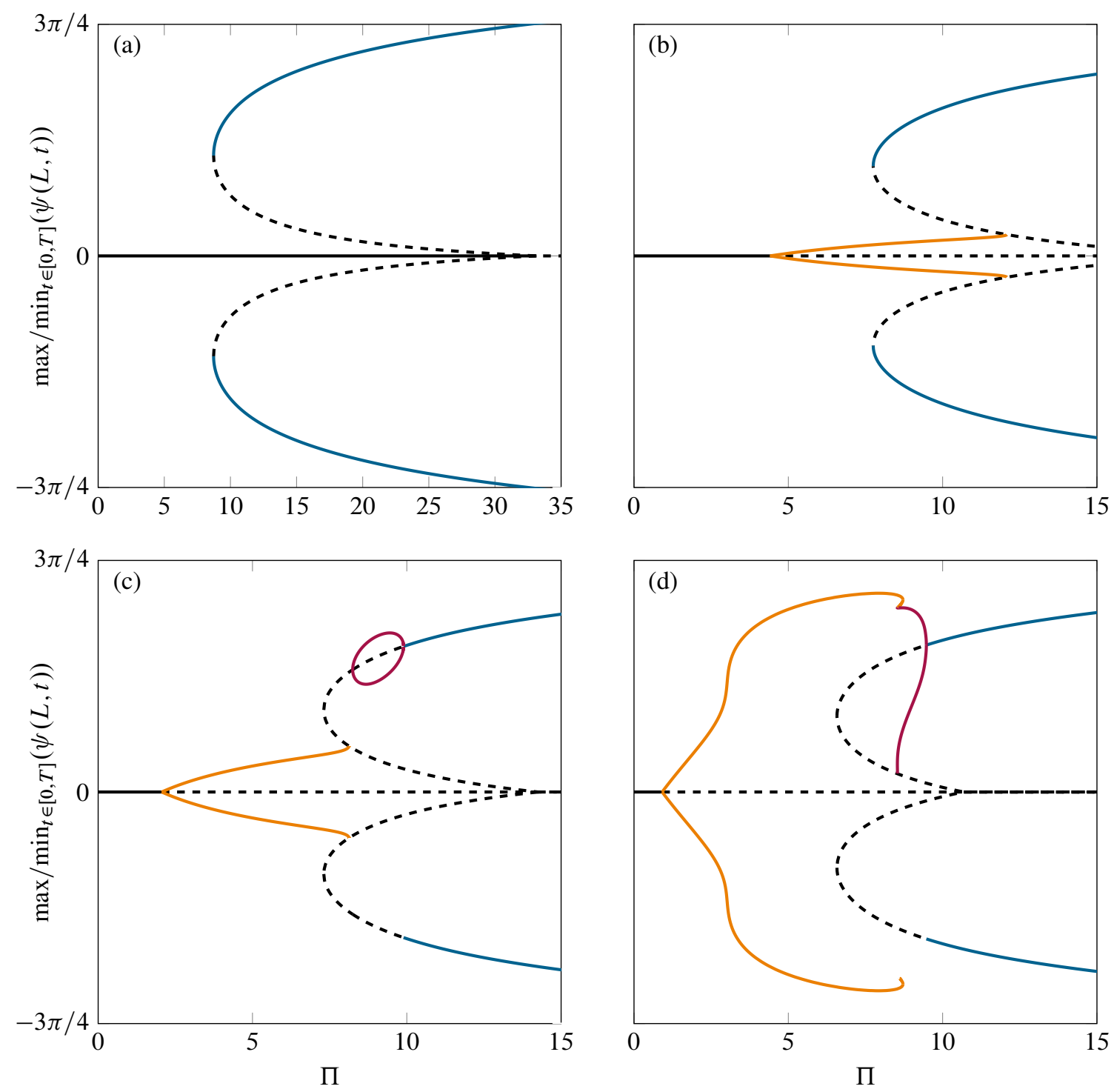

Figure 9: Bifurcation diagrams for inverted flags with $\mu=0.3$ and various aspect ratios: (a) $R=0.1$ with $k_{\mathrm{p}}=0.22$ and $k_{\mathrm{v}}=0.93$, (b) $R=0.25$ with $k_{\mathrm{p}}=0.41$ and $k_{\mathrm{v}}=0.93$ (c) $R=0.35$ with $k_{\mathrm{p}}=0.55$ and $k_{\mathrm{v}}=0.94$, and (d) $R=0.5$ with $k_{\mathrm{p}}=0.75$ and $k_{\mathrm{v}}=0.89$. Maximum/minimum values of the tip slope of the inverted flag over a cycle of steady-state oscillation of period T. [-] Stable static solution (undeflected stable equilibria), [--.] unstable static solution, [-] stable periodic solution (oscillation around undeflected equilibria), [-] stable periodic solution (oscillations around deflected equilibria), and [-] stable static solution (deflected static equilibria).

deflected equilibria emerging from a saddle-node bifurcation at $\Pi=8.6$. This behaviour agrees with other existing analytical studies $[25,31]$. One underlying reason for this is that when the aspect ratio is small, the contribution of the reactive component of the fluid forces is weak and the vortex force and viscous damping may dominate. In this case, the steady parts of the fluid forces are responsible for initiating the instability, in the form of a static divergence, also known as buckling, via a pitchfork bifurcation. Moreover, the response of the system is strongly subcritical due to the contribution of the nonlinear term in the normal force acting on the inverted flag (see Eq. (6)). More specifically, for small aspect ratios, $k_{\mathrm{v}} \rightarrow 2$ while $k_{\mathrm{p}} \rightarrow 0$. As such, the solution branches meet at relatively large $\Pi$ (e.g. $\Pi=33.1$ for $A=0.1$, Figure 9(a)) where new solutions branch off from the trivial solution and fold at a relatively small $\Pi$ (e.g. $\Pi=8.6$ for $R=0.1$ ). This results in a wide range of bistability.

As the aspect ratio becomes larger, in Figure 9(b)-9(d), the destabilizing reactive component of the fluid forces comes into play. This materializes as a supercritical Hopf bifurcation prior to the pitchfork bifurcation, thereby establishing a limit-cycle oscillation. More specifically, the Coriolis term in Eq. (15) proportional to $\mu \Pi$ generates a reaction on the inverted flag in the direction of motion. Inverted flags of sufficiently large aspect ratios undergo a large-amplitude flapping motion via a supercritical Hopf bifurcation. In particular, a Hopf bifurcation materializes at $\Pi=4.41$ preceding the saddle-node bifurcation at $\Pi=7.75$ for the flag with $R=0.25$ (Figure 9(b)), giving rise to oscillation with relatively small amplitude since $\max _{t \in[0 T]}(w(L, t))=0.08$ at $\Pi=8.0$. A range of bistability 
can be observed for $\Pi \in\left[\begin{array}{l}7.75 \\ 12.08\end{array}\right]$, wherein the response of the system could be attracted by either the stable limit cycle around the origin or the deflected equilibrium. The unstable solutions to Eq. (15) (shown as dashed lines) separate the basin of attraction of the stable limit cycle around the origin from that about the deflected equilibrium. In other words, systems with initial conditions close to the deflected equilibrium are attracted by the fixed point.

Remarkably, for sufficiently large aspect ratios, the system dynamics is more complicated. In particular, for $R=0.35$ (Figure 9(c)), the system displays a deformed-flapping motion for $\Pi \in$ [8.25 9.95]. Comparing the $A=0.35$ and $R=0.5$ cases (shown in subfigures $9(\mathrm{c})$ and $9(\mathrm{~d})$, respectively), it is seen that the deformedflapping regime narrows down and the amplitude of flapping grows faster with increasing aspect ratio. It should be realized, however, that for a true inverted flag with $R>0.2$, the large amplitude flapping takes place abruptly, and the amplitude of oscillations varies substantially with $\Pi$. To improve the results, a nonlinear analytical model accounting for the unsteady vortex shedding is called for, which is not a trivial task and virtually cannot be done in its entirety.

Moreover, the proposed model suggests that the critical flow velocity for the onset of large amplitude flapping approaches zero as $R$ increases, as a result of an increase in the magnitude of the Coriolis force (refer to the discussion in Section 3.5). This signals that the validity of the slender body model deteriorates as the aspect ratio increases.

\subsection{Influence of mass ratio}

As evidenced in Eq. (15), the mass ratio parameter $\mu$ is associated with the added mass effect and velocity-related terms. This parameter plays no role in the static solution (fixed points) but makes a significant contribution to the stability of the solution branches. More specifically, ignoring time derivatives in Eq. (15) results in a set of algebraic equations yielding the static response of the system only. In the absence of time-derivative terms in Eq. (15) or in the presence of a heavy damping, this static solution is stable prior to a pitchfork bifurcation, and it regains stability after the folding point (saddle-node bifurcation). On the other hand, introducing time-derivative terms associated with unsteady fluid forces and performing a stability analysis leads to the detection of a Hopf bifurcation.

Figure 10 compares the theoretical results obtained by the proposed model with large damping and experimental data reported by Sader et al. [25] with additional damping. The Hopf bifurcation does not occur, and hence the

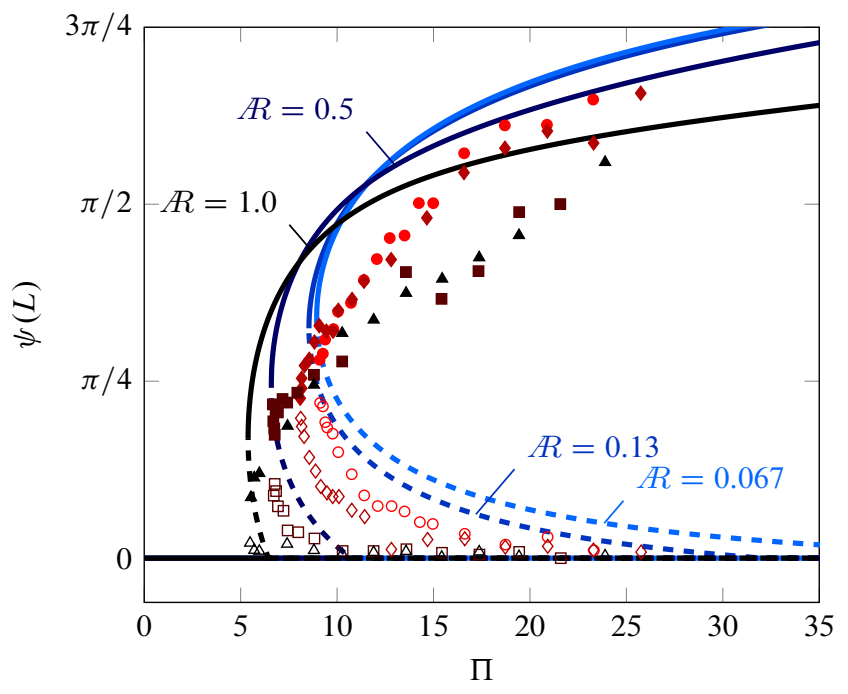

Figure 10: Comparison between static results obtained by the proposed model and experimental data reported

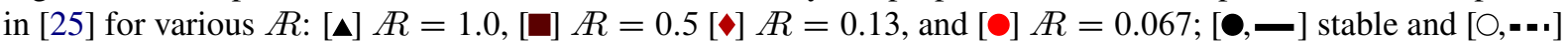
unstable deflected equilibria.

deflected equilibrium becomes stable, which agrees with the statement made just above. Interestingly, a fairly good qualitative and quantitative agreement can be seen in this figure. More specifically, Figure 10 shows a similar trend in the response calculated by the present model and the experimental results. Furthermore, it is seen that the subcritical behaviour of the response becomes weaker as $A$ increases.

Quantitative agreement may be assessed by comparing the critical flow velocity for the saddle-node bifurcation (point $\mathrm{F}$ in Figure $4(\mathrm{a})$ ). For instance, the present theory predicts $\Pi_{\mathrm{F}}=6.6$ for a slender inverted flag with $R=0.5$, whereas from the experimental data in $[25], \Pi_{\mathrm{F}} \simeq 6.7$.

On the other hand, it is evident that there is a discrepancy between theory and experiment with regard to the deflection amplitude. This is likely caused by unsteady flow forces (arising from vortex shedding) leading to a change in the mean forces on the flag, which in turn leads to a different defected equilibrium. Agreement with experiment could well improve substantially if vortex shedding were included in the model. 
Figure 11 shows the effect of $\mu$ on the nonlinear response of the inverted flag. The aim here is to investigate the relation between the length of the flag $L$ and $f$, the critical flow velocity $\Pi_{c}$ for flapping (which is also a function of $L$ ), as well as the amplitude of flapping, while the other physical parameters of the system $\left(\rho_{\mathrm{f}}, \rho_{\mathrm{p}}, h\right.$, and $\left.D\right)$ are fixed. As seen in Figure 11(a), a larger $\mu$ has the effect of generating instability at lower flow velocities, with larger
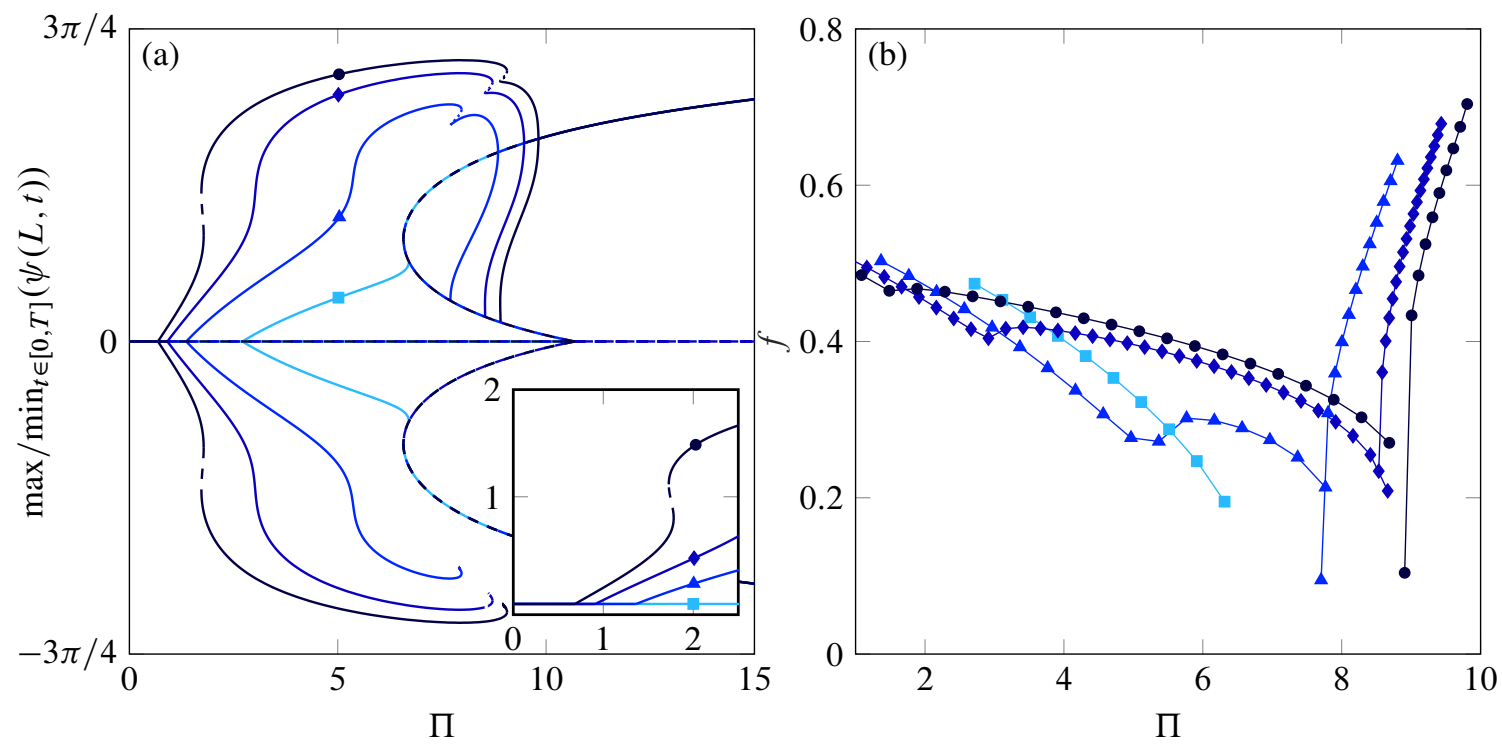

Figure 11: Mass ratio effect on the system dynamics: (a) bifurcation diagram for inverted flags with $R=0.5$ and different mass ratios: $[\square \mu=0.1[\bullet] \mu=0.2,[\mathbf{\Delta}] \mu=0.3$, and $[\mathbf{\bullet}] \mu=0.4$; (b) dimensionless frequency versus $\Pi$.

amplitude oscillations. The critical velocity precipitating flapping is inversely proportional to the mass ratio. This endorses the linear stability analysis performed by Guo \& Païdoussis [12]. The transition from the limit-cycle in the vicinity of the origin to the one in the neighbourhood of the buckled state (the deformed-flapping regime) is also affected by the mass ratio: increasing $\mu$ delays the transition between the two regimes. For instance, the saddle-node bifurcation occurs at $\Pi=7.7$ for $\mu=0.2$, while it materializes at $\Pi=8.9$ for $\mu=0.4$. The stable limit-cycle around the buckled state (in the range $\Pi \simeq 8$ to 9 for $\mu=0.2-0.4$ ) disappears for $\mu=0.1$. Interestingly, the bifurcated solution exhibits a subcritical behaviour for $\mu=0.4$, suggesting that there is hysteresis in the critical $\Pi$ obtained with decreasing and increasing flow velocity, as shown in the inset of Figure 11(a). The corresponding periodic solution loses stability via a saddle-node bifurcation at $\Pi=1.78$. The emerging unstable limit cycle folds at a second saddle-node bifurcation point $\Pi=1.72$ which corresponds to a restabilization of the periodic solution to one of slightly higher frequency and larger amplitude.

The mass ratio also affects the frequency of oscillation, as shown in Figure 11(b). For all cases, the flapping frequency decreases with the flow velocity. Furthermore, for a range of flow velocities associated with largeamplitude flapping around the undeflected equilibrium, the frequency of oscillation decreases with decreasing $\mu$. Cases with $\mu \geq 0.2$ studied here display an abrupt change in the frequency with increasing $\Pi$, corresponding to the change in the motion from large-amplitude motion around the origin to flapping around a deformed equilibrium (deformed-flapping regime).

\subsection{Strengths and limitations of the proposed model}

As with any theoretical study, the proposed model has both strengths and limitations. In this section, we address the similarities and differences in dynamical behaviour of small-aspect-ratio inverted flags as obtained via the proposed model and other theoretical/experimental studies in the literature, and we discuss the potential sources of anomalies as much as possible, putting the emphasis on physical understanding.

The measurements in [25, Figure 8] for $A=0.5$ show that the undeflected equilibrium is stable for $\Pi \lesssim 6$. This can also be inferred from figure 5 of that study which gives the critical dimensionless flow velocities for the bifurcation of the zero-deflection equilibrium. It can also be seen therein that the large-amplitude flapping is the global attractor for flow rates beyond this critical flow velocity. In other words, the inverted flag with $R=0.5$ undergoes large-amplitude flapping at circa $\Pi=6$. Furthermore, the sequence of "straight $\rightarrow$ flapping $\rightarrow$ fullydeflected" states for $R \geq 0.2$ is also available from measurements [24].

In this sense, the present results are in fair agreement with the experimental observations on the global dynamical behaviour (i.e. the type of instabilities and their sequence) of low-aspect-ratio inverted flags. Additionally, there is agreement with the 3D simulations of Tang et al. [29], predicting a similar qualitative behaviour to that in this paper for inverted flags, namely a strong sensitivity of the onset of flapping to the mass ratio parameter, signaling that the loss of stability of the trivial equilibrium is via a Hopf bifurcation. 
While comparisons above provide partial agreement between the sequence of flow regimes shown in Figure 8 of the present paper with [24, Figure 3], as well as the computational study of Tang et al.[29], we have noted a strong discrepancy between the structure of the bifurcation diagram predicted by the proposed model and that predicted via 2D simulations by Goza et al. [11] for infinitely wide inverted flags. More specifically, they identify a stable deformed equilibrium associated with a buckled configuration of the flag, emerging from a supercritical pitchfork bifurcation.

Moreover, the strong sensitivity shown to the mass ratio parameter in Figure 11 shows a rather severe disagreement with the experimental observations by Kim et al. [15], who reported no significant change in the critical flow velocity, while varying the mass ratio - although only two data points were obtained, $\mu \approx 0.4$ in air and $\mu \approx 320$ in water, and the mechanism of instability for the two systems may be of two totally distinct types, as shown by Sader et al. [24] and Goza et al. [11].

Based on the experimental observations of Kim et al. [15] on the influence of the mass ratio, Sader et al. [25, 24] employed steady-flow models and proposed a mathematical formula, connecting the critical velocity of the static saddle-node bifurcation calculated for a zero-aspect-ratio flag and that of the static divergence obtained for a flag of infinite aspect ratio. The model predicts that a flag with $R=0.5$ undergoes a static deflection at $\Pi=5.17$, which is in good agreement with measurements. Sader et al. [25] attributed this instability to the onset of flapping observed in measurements for $R=0.5$ at circa $\Pi=6$. They explained that, at this point, the flag is deflected, presenting a sharp leading edge to the impinging flow, and thus the resulting unsteady vortex shedding causes the flag to oscillate via a vortex-induced vibration mechanism.

By virtue of this conclusion, the primary limitation of the model proposed in this paper may arise from the inherent absence of unsteady vortex shedding at large angles of attack, and the approximation employed to formulate the unsteady force arising from the vortex formation along the lateral edges of the flag.

One may employ a steady-flow model, omitting the time-dependent terms in Eq. (15) (ignoring the effect of the accelerated air as it passes over and under the flag). As a result, the bifurcation diagram in Figure 4(a) gives way to a static subcritical pitchfork bifurcation, with a stable static trivial solution, as shown in Figure 12. Following

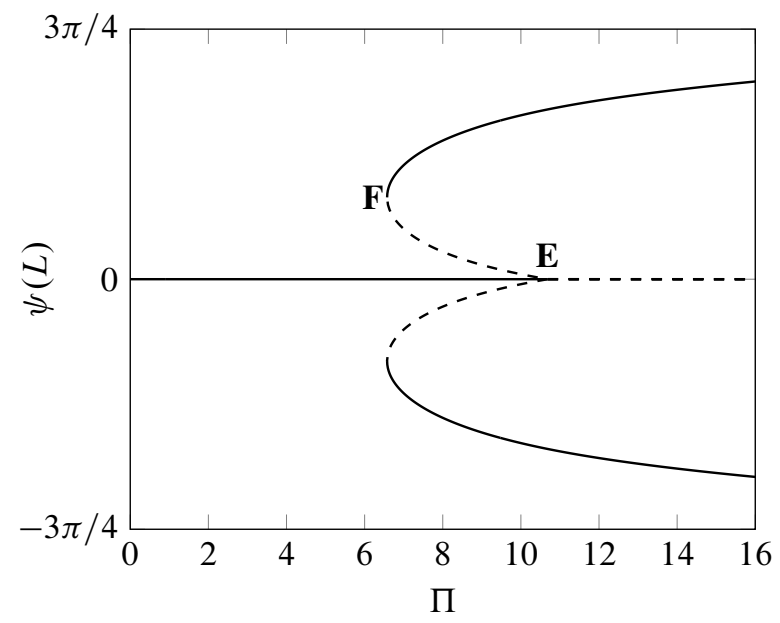

Figure 12: Bifurcation diagram of the inverted flag with $\mu=0.0$ and $R=0.5$. [-] Stable static solution (undeflected stable equilibria), [--.] unstable static solution.

Sader et al. [25], the onset of flapping can be attributed to the first instability (i.e. the static saddle-node bifurcation, marked by point $F$ ) at $\Pi=6.6$. Although a very good quantitative agreement can be found, no explanation can be proposed on how the undeflected equilibrium remains stable for velocities lower than point E; omitting unsteady forces leads to a subcritical pitchfork bifurcation for inverted flags of non-zero aspect ratio. Time-derivative terms, however, are essential in performing stability analyses on the branches of the obtained solution.

In this paper, it was found that taking into account the effect of unsteady forces - arising from the changing fluid flow momentum due to the oscillating inverted flag — causes the undeflected equilibrium to become linearly unstable. The negative damping in the system, induced by the Coriolis force, overcomes the stabilizing forces for the case of sufficiently large aspect ratio flags. The mechanism of this instability is found to be via a Hopf bifurcation with a critical velocity inversely proportional to $\mu$. Our calculations show that this instability is very marginal and weak for very small aspect-ratio flags (the imaginary part of the complex eigenfrequency of the unstable mode is very small in magnitude and could easily become positive when damping is introduced), in line with observations by Sader et al. [25]. For larger aspect ratio flags, however, the Coriolis force becomes more dominant and becomes larger with increasing flow velocity. The first mode loses stability via a Hopf bifurcation, leading to large-amplitude limit cycle oscillations. At sufficiently high flow velocities, the vortex lift force, $F_{\mathrm{R}}$, which opposes the Coriolis force, grows in magnitude, and this leads the non-trivial static solution (deflected equilibrium) to regain stability. 
The loss of stability via a Hopf bifurcation for inverted flags with $A \gtrsim 0.1$ differs from the speculation of a divergence instability proposed in $[15,24,25]$ as outlined above. A reason for that could be the limitation of using an approximation of the dissipative effect of the surrounding fluid, instead of performing a full viscous flow calculation. The approximations used in the present study to model 3D effects may not be accurate enough to fully describe the underlying mechanism of the first instability, but the similarity of predictions with those of Tang et al. [29] who used numerical methods to solve 3D Navier-Stokes equations leaves us with no definite conclusion. One possible improvement for analytical modelling of the problem, taking into account the 3D effects, may be to combine reactive and vortex-lift effects by extending Bollay's nonlinear lift theory to a plate with arbitrary curvature along its length, retaining the unsteady effects due to the shed vortices.

Further elucidation of 3D effects requires future studies.

\subsection{Sensitivity to viscous drag}

In this section, an attempt has been made to improve the fluid-forces approximated in the foregoing on viscous drag and to explore the effect on the stability and post-critical response of the system (refer to [30]). The effect of the viscous drag $k_{\tau}$ on the response is investigated numerically, and the results are shown in Figure 13. The system considered is a system with the mechanical parameters detailed in Figure 4, but with $k_{\tau}$ varied. It should be emphasized that the viscous drag is assumed to be evenly distributed along the plate and is associated with a constant viscous drag coefficient $k_{\tau}$; also, its contribution in the $d r a g$ force (in the longitudinal direction) is considered only [30].

As a first approximation for the viscous drag coefficient, the Blasius-type solution is used for both laminar and turbulent cases, that is

$$
k_{\tau}= \begin{cases}0.054 R e^{-\frac{1}{2}} & \text { for a laminar flow } \\ 1.328 R e^{-\frac{1}{7}} & \text { for a turbulent flow }\end{cases}
$$

where $R e=U L / v_{\mathrm{f}}$ denotes the Reynolds number, $v_{\mathrm{f}}$ being the fluid kinematic viscosity. For the calculation of the viscous drag modelled using Eq. (17), $R e=5 \times 10^{4}$ is considered. Furthermore, according to the measurements by Kim et al. [15], the value of the total drag (i.e., the summation of the viscous drag and the inviscid force component in the drag direction) is reported as being around 0.08 before flapping is initiated, and it jumps to around 1.0 at the critical point. The effect of this parameter is further investigated for a wider range here.

Figure 13 depicts the sensitivity to viscous drag. The effect of this parameter on the first bifurcation (where the

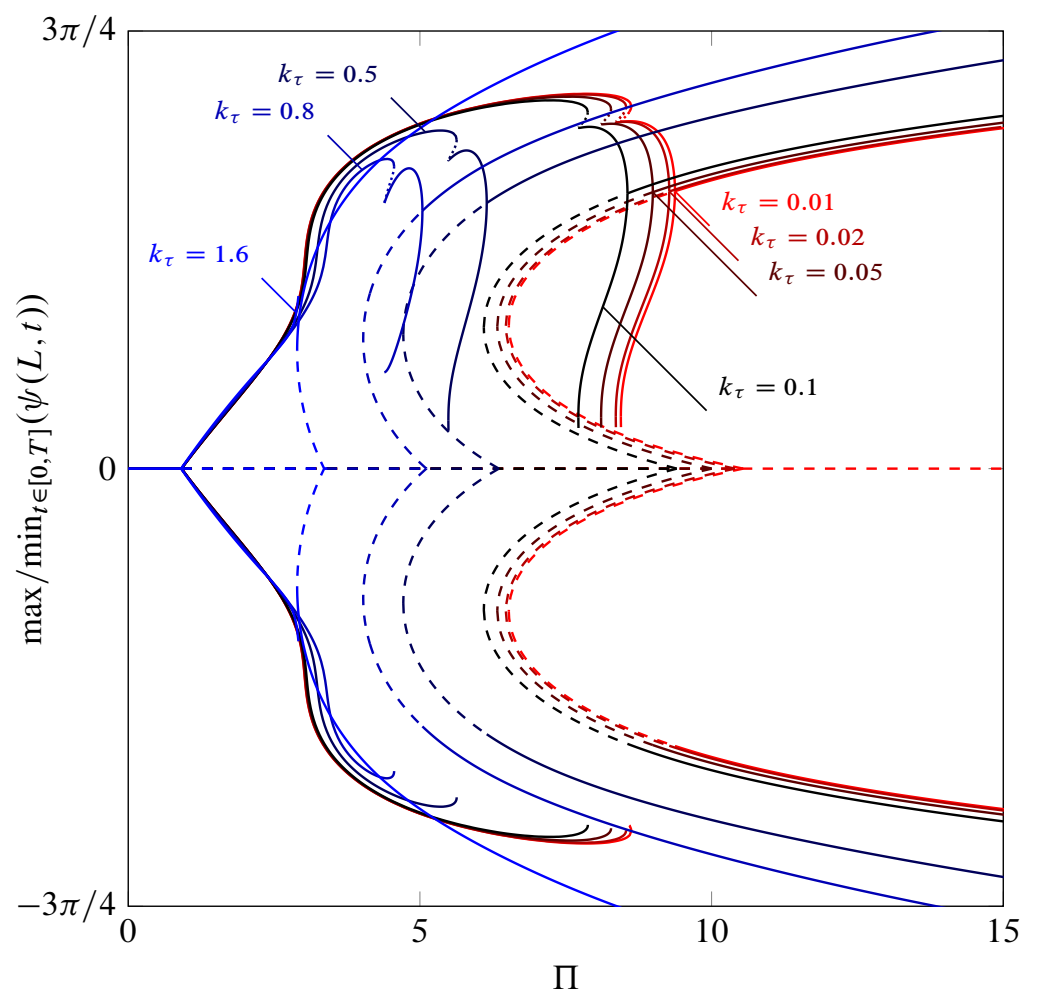

Figure 13: Bifurcation diagram of the inverted flag with $\mu=0.3$ and $R=0.5$ and various $k_{\tau}$.

stretched-straight state becomes unstable) is insignificant. However, with increasing $k_{\tau}$, subsequent bifurcations take place at lower flow velocities. In particular, the first Hopf bifurcation shifts slightly to the left in the bifurcation 
diagram, and the pitchfork bifurcation as well as the second Hopf bifurcation (where the deflected equilibrium becomes unstable) occur at smaller $\Pi$ when $k_{\tau}$ is increased. Therefore, the drag coefficient significantly affects the interval of existence of the flapping regime. Furthermore, at a given value of $\Pi$ in the flapping regime, the amplitude of oscillation decreases with increasing $k_{\tau}$. The role of the viscous drag is more significant in the range of deformed-flapping regime. This range narrows down with larger $k_{\tau}$ until it vanishes. Moreover, at a fixed flow velocity, the amplitude of the static equilibrium grows with $k_{\tau}$.

\subsection{Sensitivity to angle of incidence}

It is of interest to explore the dynamics of the system when the neutral plane of the inverted flag is not perfectly parallel to the direction of the undisturbed flow, as illustrated in Figure 14. Considering a small angle of incidence, the

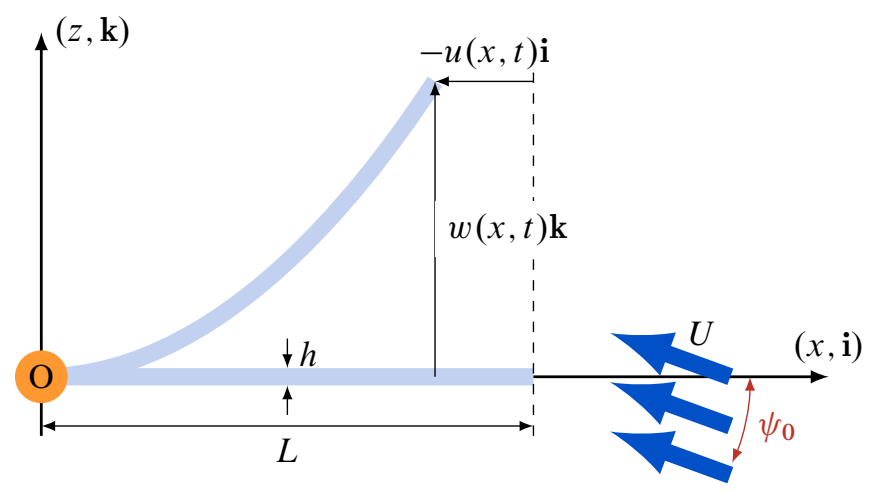

Figure 14: Inverted flag in axial flow with a small angle of incidence $\psi_{0}$

undistributed flow velocity can be decomposed into two components in the $x$ and $z$ directions as $U_{x}=-U \cos \psi_{0} \mathbf{i}$ and $U_{z}=U \sin \psi_{0} \mathbf{k}$. Consequently, the fluid-related forces should be updated in order to take into account both components. The relative velocity normal and tangential components in Eq. (2) as well as the instantaneous angle of attack in Eq. (3) become

$$
\begin{aligned}
\mathbf{V}_{\mathrm{n}} & =\left(\left(\dot{w}-U \sin \psi_{0}\right) \cos \psi-\left(\dot{u}+U \cos \psi_{0}\right) \sin \psi\right) \mathbf{n}, \\
\mathbf{V}_{\tau} & =\left(\left(\dot{u}+U \cos \psi_{0}\right) \cos \psi+\left(\dot{w}-U \sin \psi_{0}\right) \sin \psi\right) \boldsymbol{\tau}, \\
\alpha & =\psi-\operatorname{atan}\left(\frac{\dot{w}-U \sin \psi_{0}}{\dot{u}+U \cos \psi_{0}}\right) .
\end{aligned}
$$

A sensitivity analysis on the effect of the angle of incidence on the dynamics has been performed by considering four angles $\psi_{0}$. The resulting bifurcation diagrams are plotted in Figure 15. As seen in Figure 15(a), a small angle of incidence $\left(\psi_{0}=0.1\right.$, corresponding to $\left.5.7^{\circ}\right)$ breaks the symmetric response of the system, and the pitchfork bifurcation is replaced by a saddle-node bifurcation. The inverted flag exhibits an asymmetric flapping motion with a slight deviation from symmetric flapping. More interesting behaviour can be observed for larger values of $\psi_{0}$. Figure 15(b) shows the bifurcation diagram for $\psi_{0}=0.2$. Four jumps associated with four saddle-node bifurcations are observed. More specifically, increasing the flow velocity, the amplitude of oscillation increases reaching to $\max _{t \in[0 T]}(\psi(L, t))=7 \pi / 25$ at $\Pi=4$.73. At this point, the response of the system exhibits a jump and morphs into a large-amplitude limit-cycle. Further increasing the flow velocity leads to an additional jump at $\Pi=6.55$. A reverse scenario is expected when the system is initially flapping with a large amplitude and the flow velocity is decreased. The system then undergoes a jump at $\Pi=2.76$ and is attracted to the static equilibrium. The first two saddle-node bifurcations bracket a bistable region wherein the response of the system is attracted either by the stable limit cycles or the stable fixed points. The bistable region becomes narrower for larger values of $\psi_{0}$ (for instance, for $\psi_{0}=0.3$ in Figure 15(c)), and eventually is replaced by a gradual growth of oscillation amplitude (see Figure 15(d) for $\left.\psi_{0}=0.4\right)$.

In Figure 15, a small $\psi_{0}$ does not affect the large-amplitude asymmetric flapping regime much, signifying a relatively minor effect of small initial inclination on the amplitude oscillation. In particular, the peak-to-peak amplitude of the tip is 1.64 for $\psi_{0}=0.1$ and 1.57 for $\psi_{0}=0.2$, while it is 1.37 for $\psi_{0}=0.3$ and 0.76 for $\psi_{0}=0.4$. Thus, increasing $\psi_{0}$ beyond $\psi_{0}=0.3$ decreases the amplitude of asymmetric flapping significantly. In addition, the range of asymmetric flapping motions shrinks for inverted flags with larger angle of incidence, in line with the conclusions of Tang et al. [29].

Figure 16 shows the deflected or flapping shapes of the inverted flag with $\psi_{0}=0.4$ by superimposing views of the flag motion in one cycle. The trend shown agrees with previous experiments conducted by Cossé et al. [4]: flapping emerges only on one side of the inverted flag for sufficiently large angles of incidence, and the flapping amplitude decreases with $\psi_{0}$. 

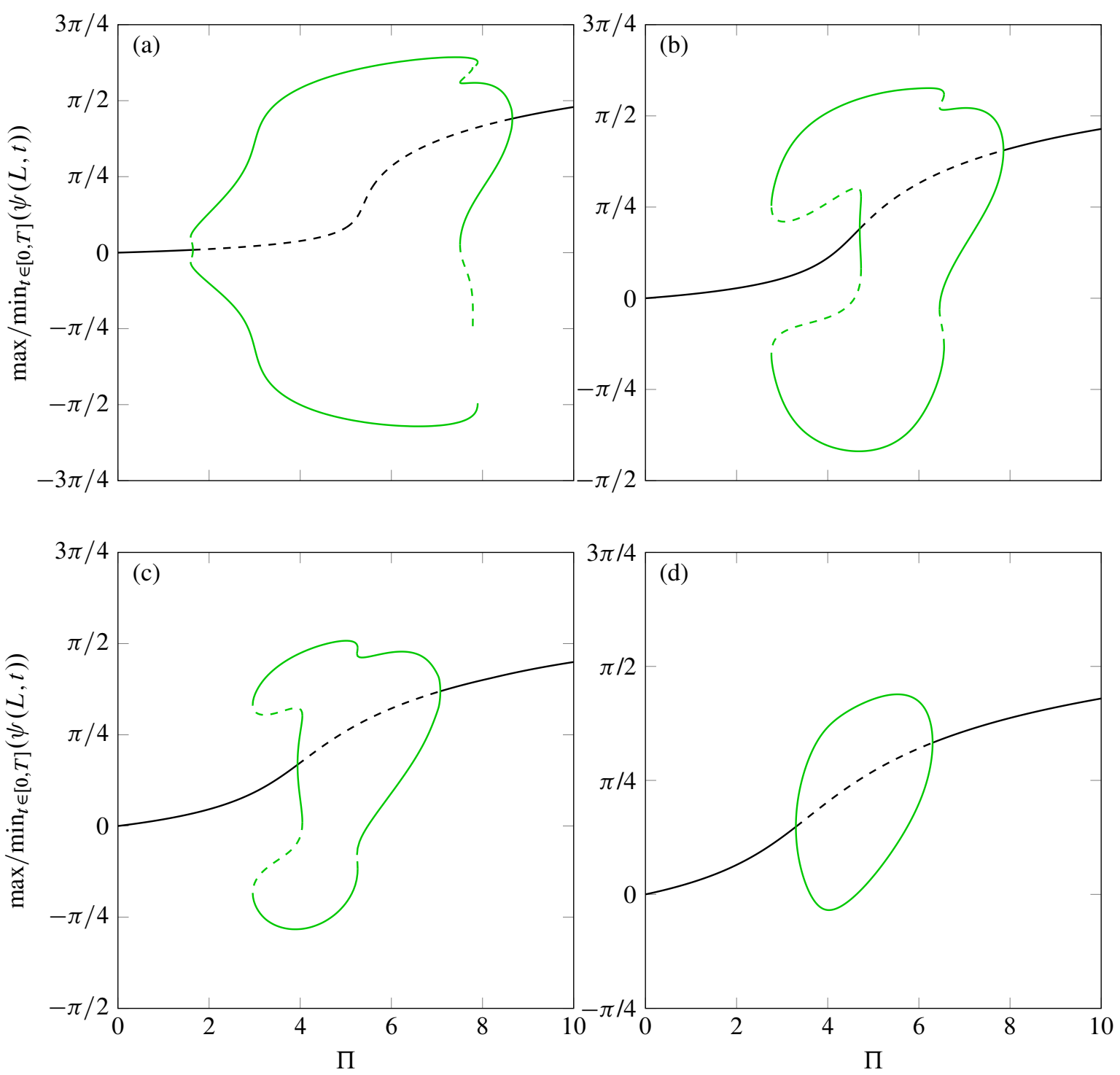

Figure 15: Bifurcation diagrams for inverted flags with $\mu=0.3, R=0.5$, and various initial angles of incidence: (a) $\psi_{0}=0.1$, (b) $\psi_{0}=0.2$, (c) $\psi_{0}=0.3$, and (d) $\psi_{0}=0.4$. Maximum/minimum values of the tip slope of the inverted flag over a cycle of steady-state oscillation of period $T$. [ - ] Stable static solution, [--.] unstable static solution and $[-]$ stable periodic solution (asymmetric oscillations).

(a)

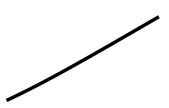

(b)

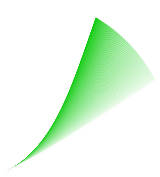

(c)

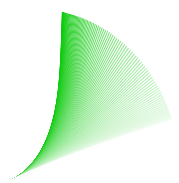

(d)

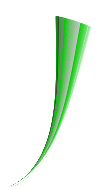

(e)

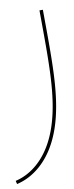

Figure 16: Superimposed deflections of an inverted flag with $\mu=0.3, R=0.5$, and $\psi_{0}=0.4$ at different flow velocities: (a) $\Pi=1.0$, (b) $\Pi=3.5$, (c) $\Pi=4.0$, and (d) $\Pi=6.2$, (e) $\Pi=10.0$.

\section{Concluding remarks}

The nonlinear response of an inverted flag (thin elastic plate) was investigated in this paper. An analytical model was developed taking into account the geometric nonlinearities and the inertia of the flag. Fluid-related forces were treated independently by employing the extension of the large-amplitude elongated-body theory accounting for reactive forces acting on the flag, as well as a vortex lift mechanism based on Bollay's model, retaining unsteady (velocity-dependent) forces. The nonlinear partial integro-differential equation was first discretized via a Galerkin technique and then solved numerically using Gear's backward differentiation formula. Bifurcation diagrams were 
obtained through pseudo-arclength continuation. The results are presented as bifurcation diagrams, time histories, phase portraits, FFT plots, and flapping shapes, so as to explore the rich dynamical behaviour of the system.

The overall nonlinear responses predicted by the analytical model exhibit several of qualitative similarities to those observed experimentally and reported in the literature. The responses exposed in this work indicate that the change in the flag aspect ratio substantially alters the dynamics. Although inverted flags of small aspect ratio do not exhibit flapping motion, inverted flags of sufficiently large aspect ratios do undergo a flapping motion. More specifically, in the case of slender inverted flags (i.e. flags of very small aspect ratio), the undeflected equilibrium loses stability via a subcritical pitchfork bifurcation giving rise to multiple additional equilibrium states. The unsteady component of the flow increases in strength with increasing aspect ratio. This results in quite intricate bifurcation mechanisms. It was seen that the undeflected equilibrium loses stability via a supercritical Hopf bifurcation in the first mode, the amplitude of which increases with the flow velocity. In particular, the inverted flag exhibits various dynamical regimes as the flow velocity is varied: (i) it initially resides at the undeflected equilibrium; (ii) it then exhibits a large-amplitude flapping motion around the undeflected equilibrium; (iii) this regime switches to a deformed-flapping motion; and finally, (iv) the system remains at the fully deflected static state with a highly curved shape.

The effect of various dimensionless parameters was investigated. It was shown that an initial angle of incidence affects the onset of flapping and the symmetry of the response. The stretched-straight state is replaced by a bent state, the amplitude of which subsequently increases with flow velocity. Increasing the angle of incidence results in a narrower range for the flapping regime, with smaller amplitude and stronger asymmetric oscillations.

It was found that flags with larger mass ratios display larger amplitude flapping and exhibit flapping at lower flow velocities.

It was also shown that the dynamical response is sensitive to viscous drag. Ignoring the viscous drag affects the response of the system considerably. In particular, it affects the range of flapping significantly and dampens the flapping motion around the deflected equilibrium.

Finally, in the present study, an attempt was made to explore the dominant physical mechanisms in the nonlinear dynamics of an inverted flag. Whereas there are a number of similarities in the results obtained here with measurements and results obtained by fully-coupled fluid-elastic simulations available in the literature, some inexplicable differences abide also. Differences were attributed to the limitations of inviscid flow theory. In particular, since the model is based on the assumption of attached flow, it is not expected to capture the aerodynamic forces beyond the angle where massive separation at the leading edge takes place. This phenomenon can cause a change in the mean forces on the flag, which in turn can lead to a different system response. The omission of unsteady fluid forces due to vortex shedding is also a strong limitation of this model. It can be said that the proposed analytical model is essentially useful for investigating the qualitative dynamics of inverted flags, and valuable for performing fast simulations to estimate the range of flow velocities wherein flapping takes place, but the results should be regarded with some caution considering the approximations employed. Improvements may be desirable in order to account for viscous effects and unsteady vortex shedding.

\section{Acknowledgments}

The authors gratefully acknowledge the financial support by the Natural Sciences and Engineering Research Council of Canada, the Solution Mining Research Institute (SMRI) and Pipeline Research Council International (PRCI).

\section{References}

[1] Ahmed Abdelbaki, Michael P. Païdoussis, and Arun Misra. "A nonlinear model for a free-clamped cylinder subjected to confined axial flow". Journal of Fluids and Structures, 2018, 80:390-404. DOI: 10.1016/j. jfluidstructs.2018.03.006.

[2] William Bollay. "A non-linear wing theory and its application to rectangular wings of small aspect ratio". ZAMM-Journal of Applied Mathematics and Mechanics, 1939, 19(1):21-35. DOI: 10.1002/zamm. 19390190103.

[3] Fabien Candelier, Frédéric Boyer, and Alban Leroyer. "Three-dimensional extension of Lighthill's largeamplitude elongated-body theory of fish locomotion". Journal of Fluid Mechanics, 2011, 674:196-226. DOI: 10.1017/S002211201000649X. HAL: hal-01145115.

[4] Julia Cossé, John Sader, Daegyoum Kim, Cecilia Huertas Cerdeira, and Morteza Gharib. "The effect of aspect ratio and angle of attack on the transition regions of the inverted flag instability". ASME 2014 Pressure Vessels and Piping Conference. Anaheim, California, 2014. DOI: 10.1115/PVP2014-28445.

[5] Adam DeVoria and Kamran Mohseni. "A vortex model for forces and moments on low-aspect-ratio wings in side-slip with experimental validation". Proceedings of the Royal Society of London A, 2017, 473(2198):20160760(1-25). DOI: 10.1098/rspa.2016.0760. 
[6] Olivier Doaré and Sébastien Michelin. "Piezoelectric coupling in energy-harvesting fluttering flexible plates: linear stability analysis and conversion efficiency". Journal of Fluids and Structures, 2011, 27(8):1357-1375. DOI: 10.1115/PVP2014-28445. arXiv: arxiv:1104.3732v1.

[7] Eusebius Doedel, Alan Champneys, Thomas Fairgrieve, Yuri Kuznetsov, Björn Sandstede, and Xianjun Wang. Continuation and Bifurcation Software for Ordinary Differential Equations (with HomCont). AUTO97, Concordia University, Canada. 1997.

[8] Christophe Eloy, Nicolas Kofman, and Lionel Schouveiler. "The origin of hysteresis in the flag instability". Journal of Fluid Mechanics, 2012, 691:583-593. DOI: 10.1017/jfm.2011.494. arXiv: arxiv:1109.4196.

[9] Charles William Gear. "The automatic integration of ordinary differential equations". Communications of the ACM, 1971, 14(3):176-179. DOI: 10.1145/362566.362571.

[10] Klaus Gersten. "A nonlinear lifting-surface theory especially for low aspect-Ratio wings". AIAA Journal, 1963, 1(4):924-925. DOI: 10.2514/3.1675.

[11] Andres Goza, Tim Colonius, and John Sader. "Global modes and nonlinear analysis of inverted-flag flapping". Journal of Fluid Mechanics, 2018, 857:312-344. DOI: 10.1017/jfm.2018.728. arXiv: arxiv:1709.09745.

[12] Chang Qin Guo and Michael P. Païdoussis. "Stability of rectangular plates with free side-edges in twodimensional inviscid channel flow". Journal of Applied Mechanics, 2000, 67(1):171-176. DOI: doi:10.1115/ 1.321143 .

[13] Pardha S. Gurugubelli and Rajeev K. Jaiman. "Large amplitude flapping of an inverted elastic foil in uniform flow with spanwise periodicity". Journal of Fluids and Structures, 2019, 90:139-163. DOI: 10.1016/j. jfluidstructs.2019.05.009.

[14] Pardha S. Gurugubelli and Rajeev K. Jaiman. "Self-induced flapping dynamics of a flexible inverted foil in a uniform flow". Journal of Fluid Mechanics, 2015, 781:657-694. DOI: 10.1017/jfm.2015.515.

[15] Daegyoum Kim, Julia Cossé, Cecilia Huertas Cerdeira, and Morteza Gharib. "Flapping dynamics of an inverted flag”. Journal of Fluid Mechanics, 2013, 736. DOI: 10.1017/jfm.2013.555. HAL: hal-02005035.

[16] Michael James Lighthill. “Aquatic animal propulsion of high hydromechanical efficiency”. Journal of Fluid Mechanics, 1970, 44(2):265-301. DOI: 10.1017/S0022112070001830.

[17] Michael James Lighthill. "Large-amplitude elongated-body theory of fish locomotion". Proceedings of the Royal Society of London B, 1971, 179(1055):125-138. DOI: 10.1098/rspb.1971.0085.

[18] Yahya Modarres-Sadeghi. "Nonlinear dynamics of a slender flexible cylinder subjected to axial flow". [pdf]. PhD thesis. McGill University, 2006.

[19] Santiago Orrego, Kourosh Shoele, Andre Ruas, Kyle Doran, Brett Caggiano, Rajat Mittal, and Sung Hoon Kang. "Harvesting ambient wind energy with an inverted piezoelectric flag". Applied Energy, 2017, 194:212222. DOI: 10.1016/j.apenergy.2017.03.016.

[20] Sung Goon Park, Boyoung Kim, Cheong Bong Chang, Jaeha Ryu, and Hyung Jin Sung. "Enhancement of heat transfer by a self-oscillating inverted flag in a Poiseuille channel flow". International Journal of Heat and Mass Transfer, 2016, 96:362-370. DOI: 10.1016/j.ijheatmasstransfer.2016.01.043.

[21] Michael P. Païdoussis. Fluid-Structure Interactions: Slender Structures and Axial Flow. 2nd ed. Vol. 1. Oxford: Academic Press, 2014.

[22] Michael P. Païdoussis, Phuong Luu, and Sairam Prabhakar. "Dynamics of a long tubular cantilever conveying fluid downwards, which then flows upwards around the cantilever as a confined annular flow". Journal of Fluids and Structures, 2008, 24(1):111-128. DOI: 10.1016/j.jfluidstructs.2007.07.004.

[23] Edward Polhamus. "A concept of the vortex lift of sharp-edge delta wings based on a leading-edge-suction analogy”. NASA Technical Note, 1966. URL: http://hdl.handle.net/2060/19670003842.

[24] John Sader, Julia Cossé, Daegyoum Kim, Boyu Fan, and Morteza Gharib. "Large-amplitude flapping of an inverted flag in a uniform steady flow - a vortex-induced vibration". Journal of Fluid Mechanics, 2016, 793:524-555. DOI: 10.1017/jfm.2016.139.

[25] John Sader, Cecilia Huertas-Cerdeira, and Morteza Gharib. "Stability of slender inverted flags and rods in uniform steady flow”. Journal of Fluid Mechanics, 2016, 809:873-894. DOI: 10.1017/jfm.2016.691.

[26] Lionel Schouveiler, Christophe Eloy, and Patrice Le Gal. "Flow-induced vibrations of high mass ratio flexible filaments freely hanging in a flow”. Physics of Fluids, 2005, 17(4):047104(1-8). DOI: 10.1063/1.1878292. HAL: hal-00014491.

[27] Christian Semler, Guang Xuan Li, and Michael P. Païdoussis. "The non-linear equations of motion of pipes conveying fluid”. Journal of Sound and Vibration, 1994, 169(5):577-599. DOI: 10.1006/jsvi.1994.1035.

[28] Kourosh Shoele and Rajat Mittal. "Flutter instability of a thin flexible plate in a channel". Journal of Fluid Mechanics, 2016, 786:29-46. DOI: 10.1017/jfm.2015.632. 
[29] Chao Tang, Nan-Sheng Liu, and Xi-Yun Lu. "Dynamics of an inverted flexible plate in a uniform flow". Physics of Fluids, 2015, 27(7). DOI: 10.1063/1.4923281.

[30] Liaosha Tang and Michael P. Païdoussis. "On the instability and the post-critical behaviour of two-dimensional cantilevered flexible plates in axial flow". Journal of Sound and Vibration, 2007, 305(1-2):97-115. DOI: 10.1016/j.jsv.2007.03.042.

[31] Mohammad Tavallaeinejad, Michael P. Païdoussis, and Mathias Legrand. "Nonlinear static response of lowaspect-ratio inverted flags subjected to a steady flow". Journal of Fluids and Structures, 2018, 83:413-428. DOI: 10.1016/j.jfluidstructs.2018.09.003. HAL: hal-01745147.

[32] Geoffrey Taylor. “Analysis of the swimming of long and narrow animals". Proceedings of the Royal Society of London A, 1952, 214(1117):158-183. DOI: 10.1098/rspa.1952.0159.

[33] Gabriel Torres and Thomas Mueller. "Aerodynamic characteristics of low aspect ratio wings at low Reynolds numbers, fixed and flapping wing aerodynamics for micro air vehicle applications". Progress in Astronautics and Aeronautics, 2001, 195:115-141. DOI: 10.2514/5.9781600866654.0115.0141.

[34] Yuri Yadykin, Victor Tenetov, and Daniel Levin. "The flow-induced vibration of a flexible strip hanging vertically in a parallel flow. Part 1: Temporal aeroelastic instability". Journal of Fluids and Structures, 2001, 15(8):1167-1185. DOI: 10.1006/jfls.2001.0400.

[35] Yuelong Yu, Yingzheng Liu, and Yujia Chen. "Vortex dynamics and heat transfer behind self-oscillating inverted flags of various lengths in channel flow". Physics of Fluids, 2018, 30(4):045104(1-14). DOI: 10.1063/1.5022723.

[36] Yuelong Yu, Yingzheng Liu, and Yujia Chen. "Vortex dynamics behind a self-oscillating inverted flag placed in a channel flow: Time-resolved particle image velocimetry measurements". Physics of Fluids, 2017, 29(12):125104(1-15). DOI: 10.1063/1.5001967.

[37] Zhanle Yu and Christophe Eloy. "Extension of Lighthill's slender-body theory to moderate aspect ratios". Journal of Fluids and Structures, 2018, 76:84-94. DOI: 10.1016/j.jfluidstructs.2017.09.010. HAL: hal01765397. 